\title{
Automatic Adaptive Sampling in Parametric Model Order Reduction by Matrix Interpolation
}

Maria Cruz Varona*, Mashuq-un-Nabi ${ }^{\ddagger}$, Boris Lohmann§

* Chair of Automatic Control,

Technical University of Munich, Germany

‡Department of Electrical Engineering,

Indian Institute of Technology Delhi, India

July 4th, AIM 2017

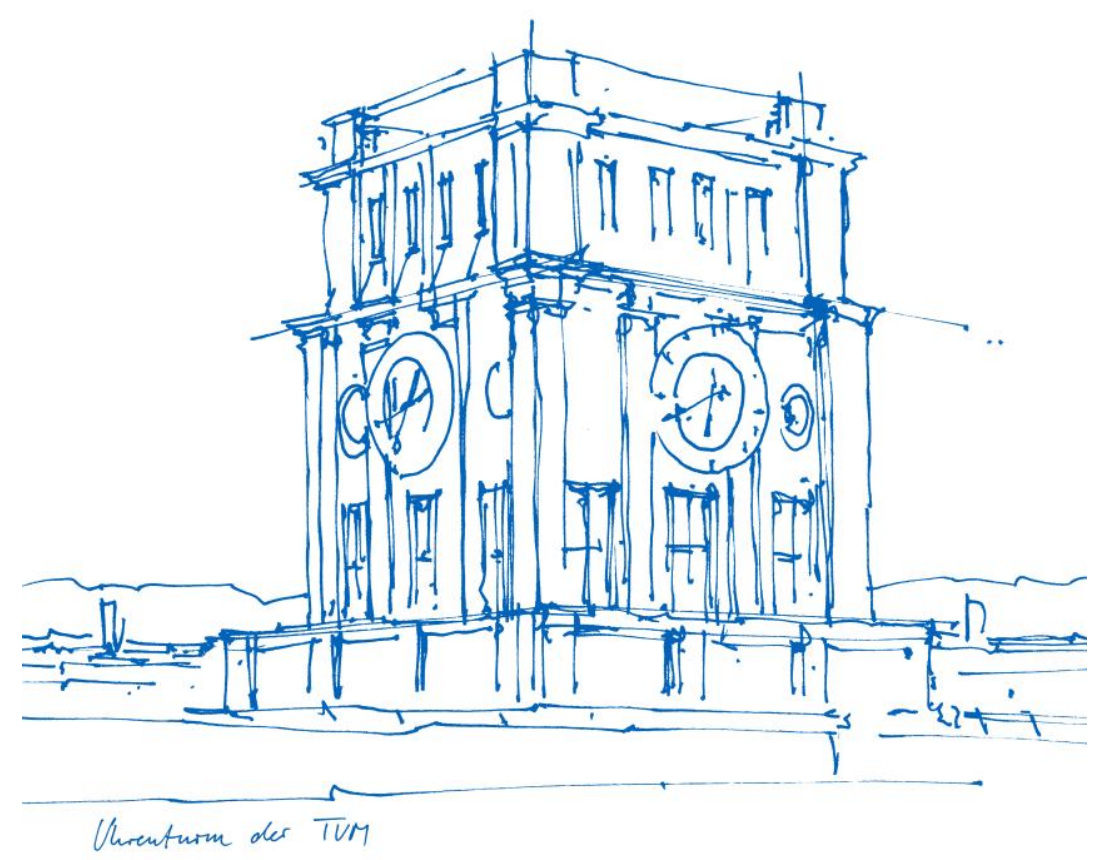




\section{Motivation}

Large-scale full order model (FOM)
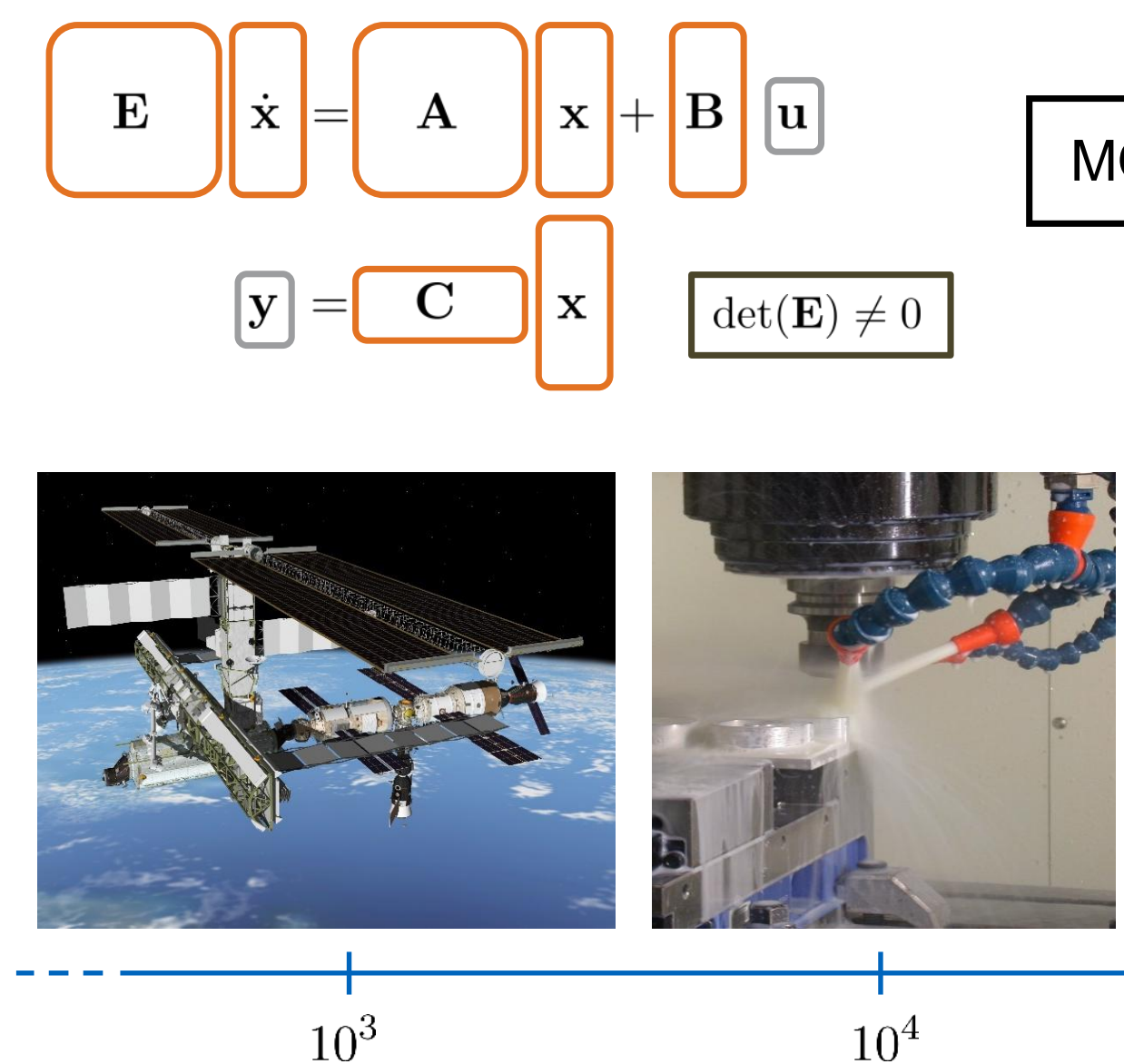

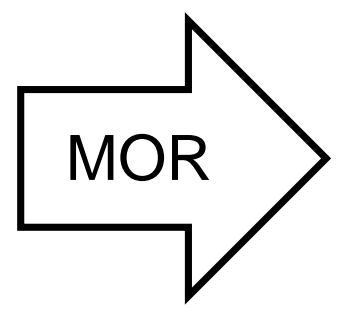

$\mathbf{E}_{r}$

$$
\mathbf{y}_{r}=\mathbf{C}_{r} \mathbf{x}_{r}
$$

$$
\mathbf{x}_{r} \in \mathbb{R}^{r}, r \ll n
$$
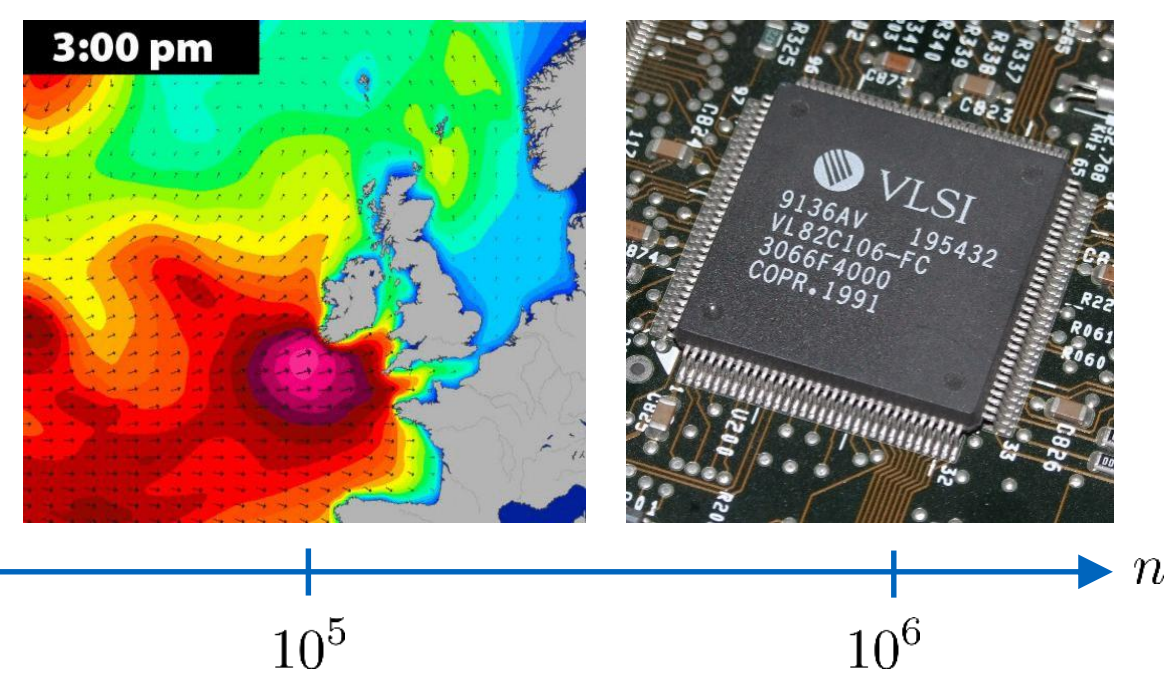


\section{Projective Model Order Reduction}

Approximation of the state vector:

$$
\mathrm{x}=\mathrm{V} \mathbf{\mathrm { x }}_{r}+\mathrm{e}, \quad \mathrm{V} \in \mathbb{R}^{n \times r}
$$

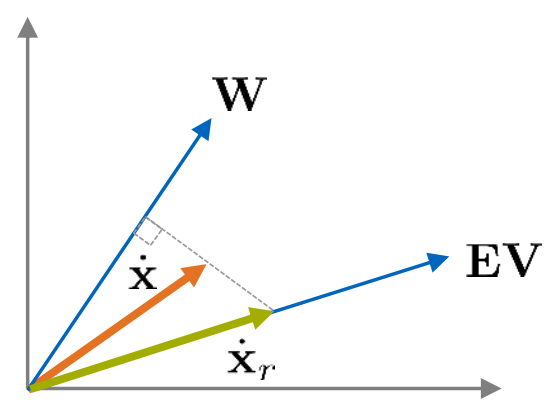

Petrov-Galerkin projection:

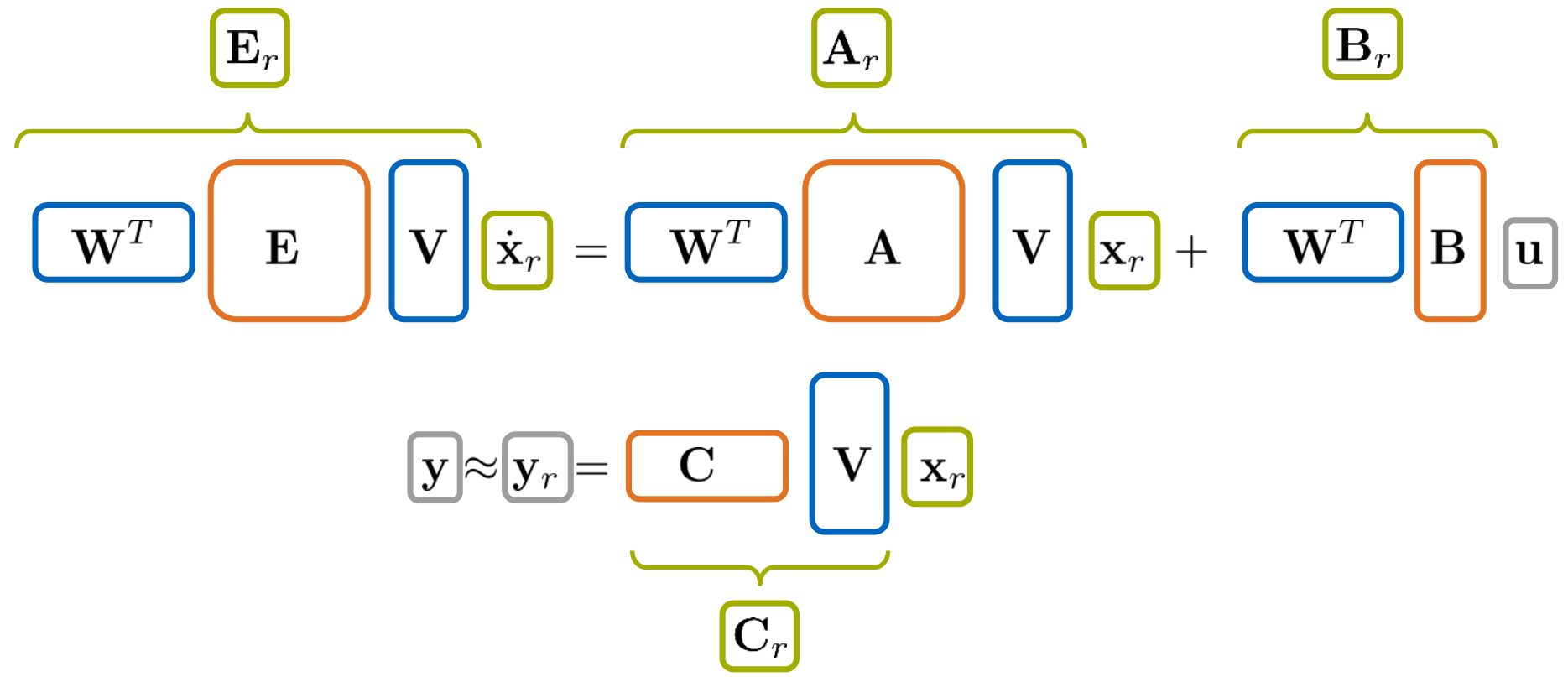




\section{Rational Interpolation by Krylov subspace methods}

Moments of a transfer function

$$
\begin{aligned}
\mathbf{G}(s) & =\mathbf{C}(s \mathbf{E}-\mathbf{A})^{-1} \mathbf{B} \\
& =\mathbf{G}\left(\Delta s+s_{0}\right)=-\sum_{i=0}^{\infty} \mathbf{M}_{i}\left(s_{0}\right)\left(s-s_{0}\right)^{i}
\end{aligned}
$$

$\mathbf{M}_{i}\left(s_{0}\right)$ : i-th moment around $s_{0}$

Krylov subspace:

$\mathcal{K}_{r}(\mathbf{M}, \mathbf{v})=\left[\mathbf{v}, \mathbf{M} \mathbf{v}, \mathbf{M}^{2} \mathbf{v}, \cdots, \mathbf{M}^{r-1} \mathbf{v}\right]$

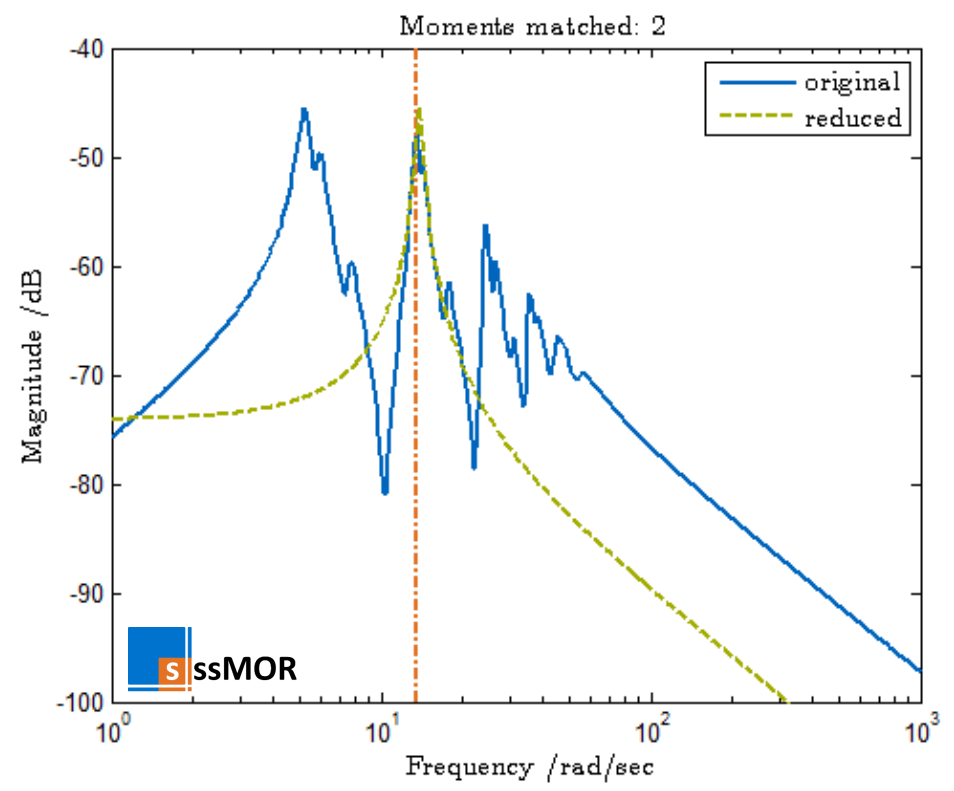

Moment Matching by Rational Krylov (RK) subspaces

Bases for input and output Krylov-subspaces:

$$
\begin{aligned}
& \operatorname{Im}(\mathbf{V})=\left[\begin{array}{llll}
\mathbf{A}_{s_{0}}^{-1} \mathbf{B}, & \mathbf{A}_{s_{0}}^{-1} \mathbf{E} \mathbf{A}_{s_{0}}^{-1} \mathbf{B}, & \ldots, & \left(\mathbf{A}_{s_{0}}^{-1} \mathbf{E}\right)^{r-1} \mathbf{A}_{s_{0}}^{-1} \mathbf{B}
\end{array}\right] \\
& \operatorname{Im}(\mathbf{W})=\left[\begin{array}{llll}
\mathbf{A}_{s_{0}}^{-T} \mathbf{C}^{T}, & \mathbf{A}_{s_{0}}^{-T} \mathbf{E}^{T} \mathbf{A}_{s_{0}}^{-T} \mathbf{C}^{T}, \quad \ldots, \quad\left(\mathbf{A}_{s_{0}}^{-T} \mathbf{E}^{T}\right)^{r-1} \mathbf{A}_{s_{0}}^{-T} \mathbf{C}^{T}
\end{array}\right] \\
& \sqsubseteq \frac{\mathbf{M}_{i}\left(s_{0}\right)=\mathbf{M}_{r, i}\left(s_{0}\right)}{\text { for } i=0, \ldots, 2 r}
\end{aligned}
$$

Moments from full and reduced order model around certain shifts match! 


\section{Parametric Model Order Reduction (pMOR)}

Large-scale parametric model

$$
\begin{aligned}
\mathbf{E}(\mathrm{p}) \dot{\mathbf{x}} & =\mathbf{A}(\mathrm{p}) \mathbf{x}+\mathbf{B}(\mathrm{p}) \mathbf{u} \\
\mathbf{y} & =\mathbf{C}(\mathrm{p}) \mathbf{x} \quad \mathbf{p} \in \mathcal{D} \subset \mathbb{R}^{d}
\end{aligned}
$$

Reduced order parametric model

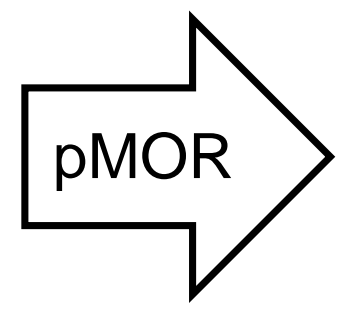

$$
\begin{aligned}
\mathbf{E}_{r}(\mathrm{p}) \dot{\mathbf{x}}_{r} & =\mathbf{A}_{r}(\mathrm{p}) \mathbf{x}_{r}+\mathbf{B}_{r}(\mathrm{p}) \mathbf{u} \\
\mathbf{y}_{r} & =\mathbf{C}_{r}(\mathrm{p}) \mathbf{x}_{r} \\
& \mathbf{x}_{r} \in \mathbb{R}^{r}, r \ll n
\end{aligned}
$$

- Linear dynamic systems with design parameters (e.g. material / geometry parameters,...)

- Goal: numerically efficient reduction with preservation of the parameter dependency

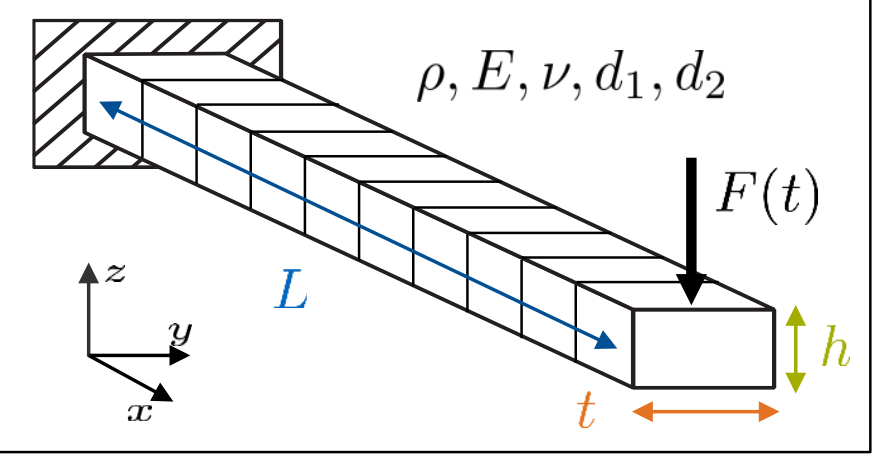

Timoshenko beam

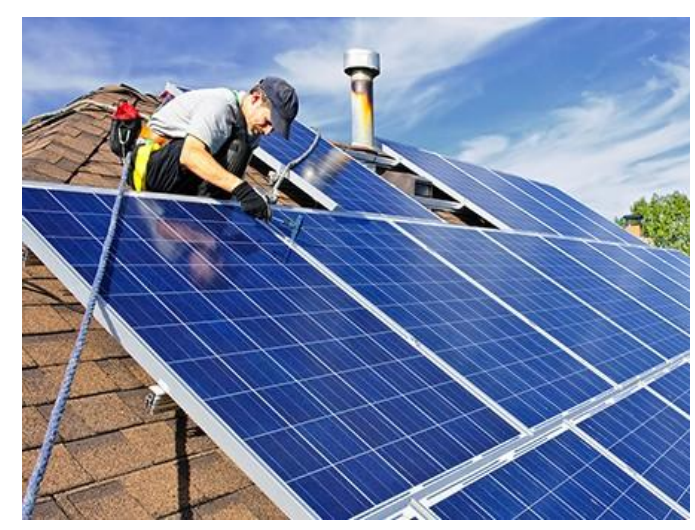

Solar panels

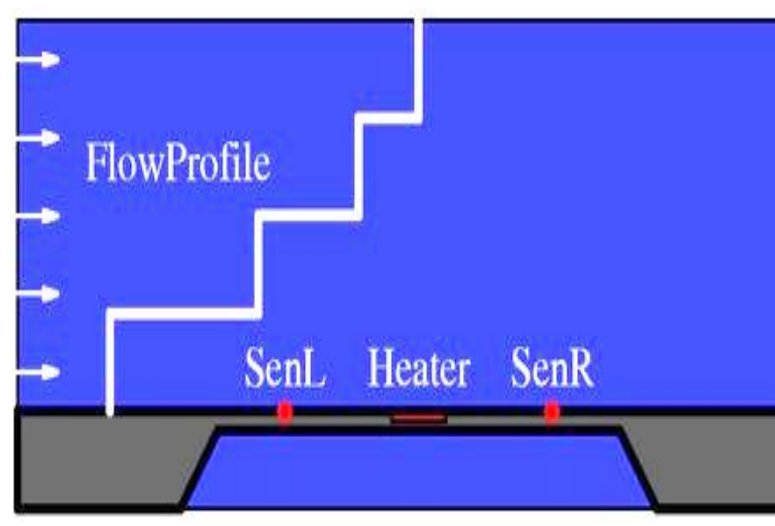

Flow sensing anemometer 


\section{pMOR by Matrix Interpolation - Main Idea}

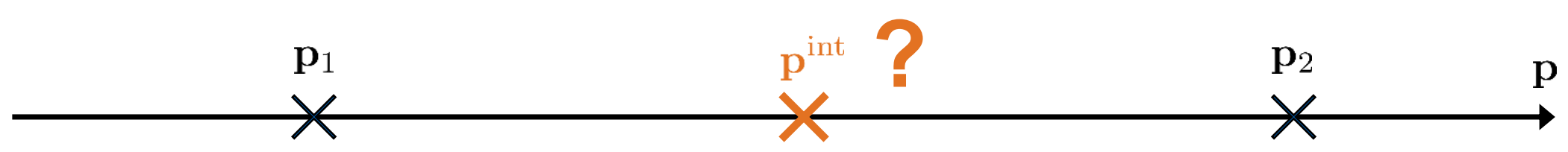

Model at $\mathbf{p}_{1}$

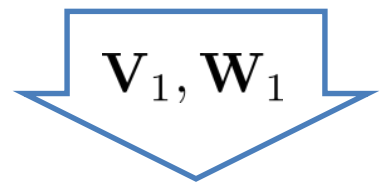

Reduced model at $\mathbf{p}_{1}$

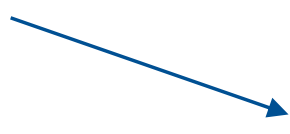

Model at $\mathbf{p}^{\text {int }}$

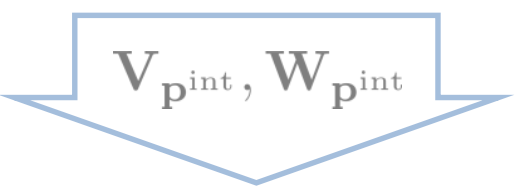

Reduced model at $\mathbf{p}^{\text {int }}$

Interpolation of

reduced matrices
Model at $\mathbf{p}_{2}$

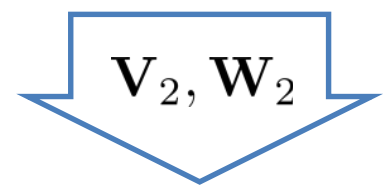

Reduced model at $\mathbf{p}_{2}$

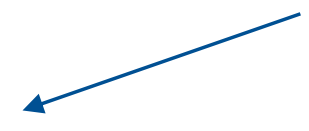

Reduced model at $\mathbf{p}^{\text {int }}$ from reduced models at sample points $\mathbf{p}_{1} \& \mathbf{p}_{2}$ 


\section{pMOR by Matrix Interpolation - Procedure}

[Panzer et al. '10]

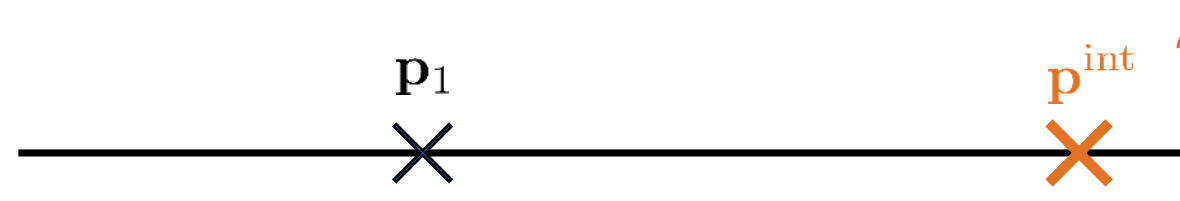

$$
\begin{aligned}
\mathbf{E}_{1} \dot{\mathbf{x}} & =\mathbf{A}_{1} \mathbf{x}+\mathbf{B}_{1} \mathbf{u} \\
\mathbf{y} & =\mathbf{C}_{1} \mathbf{x} \\
\mathbf{V}_{1}, \mathbf{W}_{1} & \\
\mathbf{E}_{r, 1} \dot{\mathbf{x}}_{r, 1} & =\mathbf{A}_{r, 1} \mathbf{x}_{r, 1}+\mathbf{B}_{r, 1} \mathbf{u} \\
\mathbf{y}_{r, 1} & =\mathbf{C}_{r, 1} \mathbf{x}_{r, 1}
\end{aligned}
$$

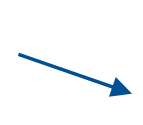

direct interpolation

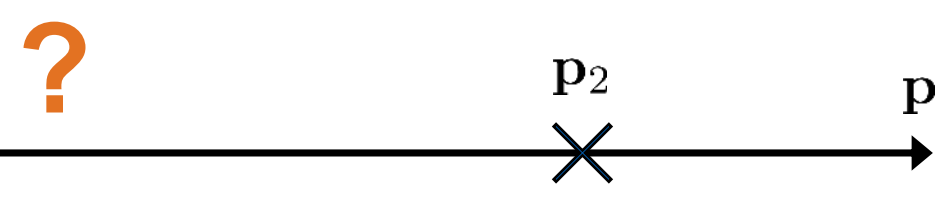
not meaningful

\section{1.) Individual reduction}

$$
\begin{aligned}
\mathbf{E}_{r, i} \dot{\mathbf{x}}_{r, i}(t) & =\mathbf{A}_{r, i} \mathbf{x}_{r, i}(t)+\mathbf{B}_{r, i} \mathbf{u}(t) & \mathbf{E}_{r, i}=\mathbf{W}_{i}^{T} \mathbf{E}_{i} \mathbf{V}_{i}, & \mathbf{A}_{r, i}=\mathbf{W}_{i}^{T} \mathbf{A}_{i} \mathbf{V}_{i} \\
\mathbf{y}_{r, i}(t) & =\mathbf{C}_{r, i} \mathbf{x}_{r, i}(t) & \mathbf{B}_{r, i}=\mathbf{W}_{i}^{T} \mathbf{B}_{i}, & \mathbf{C}_{r, i}=\mathbf{C}_{i} \mathbf{V}_{i}
\end{aligned}
$$

$\mathbf{p}_{i}, i=1, \ldots, K$

$\mathbf{V}_{i}:=\mathbf{V}\left(\mathbf{p}_{i}\right)$

$\mathbf{W}_{i}:=\mathbf{W}\left(\mathbf{p}_{i}\right)$ 


\section{pMOR by Matrix Interpolation - Procedure}

[Panzer et al. '10]

\section{1.) Individual reduction}

$$
\begin{aligned}
& \mathbf{E}_{r, i} \dot{\mathbf{x}}_{r, i}(t)=\mathbf{A}_{r, i} \mathbf{x}_{r, i}(t)+\mathbf{B}_{r, i} \mathbf{u}(t) \\
& \mathbf{E}_{r, i}=\mathbf{W}_{i}^{T} \mathbf{E}_{i} \mathbf{V}_{i}, \\
& \mathbf{A}_{r, i}=\mathbf{W}_{i}^{T} \mathbf{A}_{i} \mathbf{V}_{i} \\
& \mathbf{y}_{r, i}(t)=\mathbf{C}_{r, i} \mathbf{x}_{r, i}(t) \\
& \mathbf{B}_{r, i}=\mathbf{W}_{i}^{T} \mathbf{B}_{i} \\
& \mathbf{C}_{r, i}=\mathbf{C}_{i} \mathbf{V}_{i}
\end{aligned}
$$

$\mathbf{p}_{i}, i=1, \ldots, K$

$\mathbf{V}_{i}:=\mathbf{V}\left(\mathbf{p}_{i}\right)$

$\mathbf{W}_{i}:=\mathbf{W}\left(\mathbf{p}_{i}\right)$

\section{2.) Transformation to generalized coordinates}

$$
\begin{aligned}
\overbrace{\mathbf{M}_{i}^{T} \mathbf{E}_{r, i} \mathbf{T}_{i} \dot{\mathbf{x}}_{r, i}^{*}(t)}^{\mathbf{E}_{r, i}^{*}} & =\overbrace{\mathbf{y}_{r, i}(t)}=\underbrace{\mathbf{M}_{r, i}^{T} \mathbf{A}_{r, i} \mathbf{T}_{i} \mathbf{x}_{r, i}^{*}(t)}_{\mathbf{C}_{r, i}^{*}} \mathbf{x}_{r, i}^{*}(t)+\overbrace{\mathbf{M}_{i}^{T} \mathbf{B}_{r, i}^{*} \mathbf{u}(t)}^{\mathbf{B}_{r, i}^{*}}
\end{aligned}
$$

$$
\mathbf{x}_{r, i}=\mathbf{T}_{i} \mathbf{x}_{r, i}^{*}
$$

$$
\begin{aligned}
\mathbf{T}_{i} & =\left(\mathbf{R}^{T} \mathbf{V}_{i}\right)^{-1} \\
\mathbf{M}_{i} & =\left(\mathbf{R}^{T} \mathbf{W}_{i}\right)^{-1}
\end{aligned}
$$

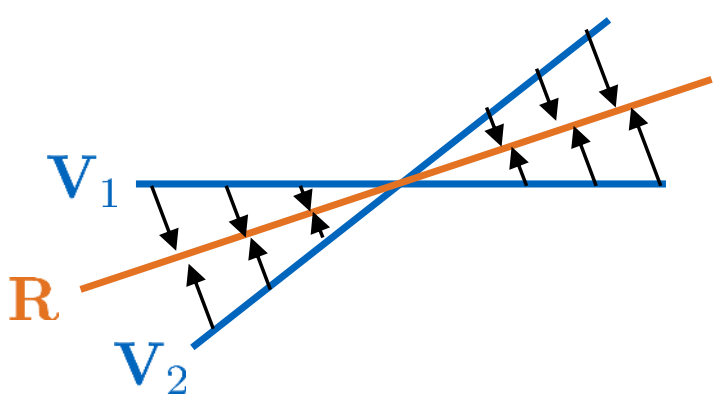

High correlation $\mathbf{V}_{i}^{*} \longleftrightarrow \mathbf{R}:$ $\mathbf{T}_{i}^{T} \mathbf{V}_{i}^{T} \mathbf{R} \stackrel{!}{=} \mathbf{I}$

Dual procedure for the local bases $\mathbf{W}_{i}$ 


\section{pMOR by Matrix Interpolation - Procedure}

[Panzer et al. '10]

1.) Individual reduction

$$
\begin{aligned}
\mathbf{E}_{r, i} \dot{\mathbf{x}}_{r, i}(t) & =\mathbf{A}_{r, i} \mathbf{x}_{r, i}(t)+\mathbf{B}_{r, i} \mathbf{u}(t) & \mathbf{E}_{r, i}=\mathbf{W}_{i}^{T} \mathbf{E}_{i} \mathbf{V}_{i}, & \mathbf{A}_{r, i}=\mathbf{W}_{i}^{T} \mathbf{A}_{i} \mathbf{V}_{i} \\
\mathbf{y}_{r, i}(t) & =\mathbf{C}_{r, i} \mathbf{x}_{r, i}(t) & \mathbf{B}_{r, i}=\mathbf{W}_{i}^{T} \mathbf{B}_{i}, & \mathbf{C}_{r, i}=\mathbf{C}_{i} \mathbf{V}_{i}
\end{aligned}
$$

$$
\begin{aligned}
& \mathbf{p}_{i}, i=1, \ldots, K \\
& \mathbf{V}_{i}:=\mathbf{V}\left(\mathbf{p}_{i}\right) \\
& \mathbf{W}_{i}:=\mathbf{W}\left(\mathbf{p}_{i}\right)
\end{aligned}
$$

\section{2.) Transformation to generalized coordinates}

$$
\begin{aligned}
& \overbrace{\mathbf{M}_{i}^{T} \mathbf{E}_{r, i} \mathbf{T}_{i}}^{\mathbf{E}_{r, i}^{*}} \dot{\mathbf{x}}_{r, i}^{*}(t)=\overbrace{\mathbf{M}_{i}^{T} \mathbf{A}_{r, i} \mathbf{T}_{i} \mathbf{x}_{r, i}^{*}(t)}^{\mathbf{A}_{r, i}^{*}}+\overbrace{\mathbf{M}_{i}^{T} \mathbf{B}_{r, i}}^{\mathbf{B}_{r, i}^{*}} \mathbf{u}(t) \\
& \mathbf{y}_{r, i}(t)=\underbrace{\mathbf{C}_{r, i} \mathbf{T}_{i}}_{\mathbf{C}_{r, i}^{*}} \mathbf{x}_{r, i}^{*}(t)
\end{aligned}
$$

$$
\mathbf{x}_{r, i}=\mathbf{T}_{i} \mathbf{x}_{r, i}^{*}
$$

$\mathbf{V}_{\text {all }}=\left[\mathbf{V}_{1}, \ldots, \mathbf{V}_{K}\right]$

$\mathbf{V}_{\text {all }} \stackrel{\text { SVD }}{=} \mathbf{U} \boldsymbol{\Sigma} \mathbf{N}^{T}$

$\mathbf{R}=\mathbf{U}(:, 1: r)$

\section{3.) Interpolation}

$$
\begin{array}{ll}
\mathbf{E}_{r}^{*}\left(\mathbf{p}^{\text {int }}\right)=\sum_{i=1}^{K} \omega_{i}(\mathbf{p}) \mathbf{E}_{r, i}^{*}, & \mathbf{A}_{r}^{*}\left(\mathbf{p}^{\text {int }}\right)=\sum_{i=1}^{K} \omega_{i}\left(\mathbf{p}^{\mathrm{int}}\right) \mathbf{A}_{r, i}^{*} \\
\mathbf{B}_{r}^{*}\left(\mathbf{p}^{\text {int }}\right)=\sum_{i=1}^{K} \omega_{i}(\mathbf{p}) \mathbf{B}_{r, i}^{*}, & \mathbf{C}_{r}^{*}\left(\mathbf{p}^{\mathrm{int}}\right)=\sum_{i=1}^{K} \omega_{i}\left(\mathbf{p}^{\mathrm{int}}\right) \mathbf{C}_{r, i}^{*}
\end{array}
$$

$\sum_{i=1}^{K} \omega_{i}\left(\mathbf{p}^{\mathrm{int}}\right)=1$

But: how to choose the sample points?? 


\section{Adaptive Sampling}

\section{Requirements:}

- Parametric space should be adequately sampled

- Avoid undersampling and oversampling

- More parameter samples should be placed in highly sensitive zones

\section{Quantification of parametric sensitivity:}

- System-theoretic measure that quantifies the parametric sensitivity is needed in order to guide the adaptive refinement

- Adaptive sampling using angle between subspaces
Uniform Sampling:
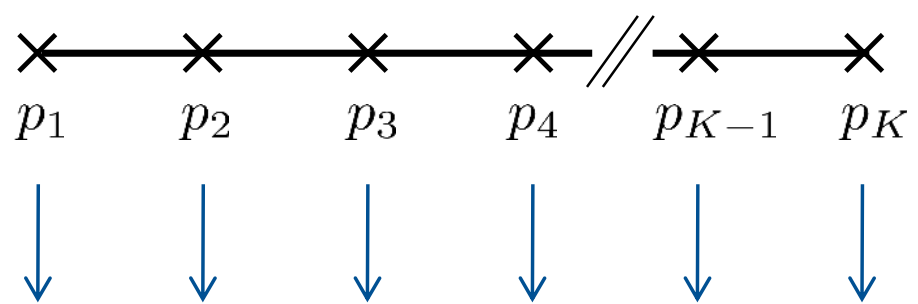

$\begin{array}{llllll}\mathbf{V}_{1} & \mathbf{V}_{2} & \mathbf{V}_{3} & \mathbf{V}_{4} & \mathbf{V}_{K-1} & \mathbf{V}_{K}\end{array}$

Adaptive Sampling:

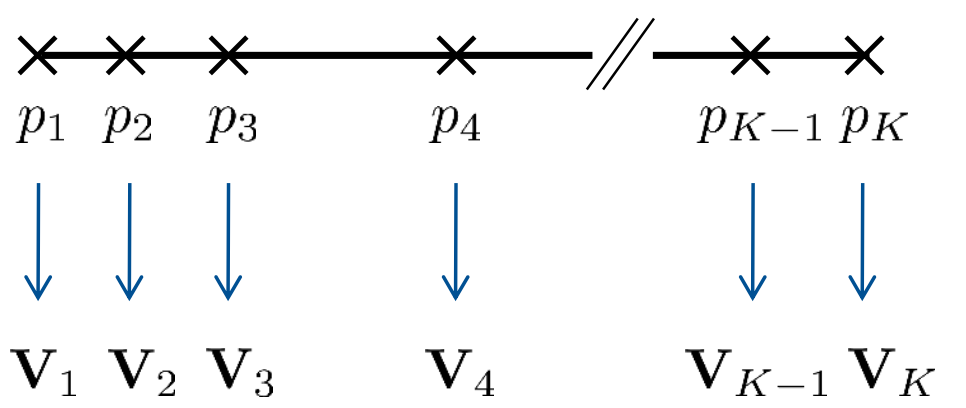




\section{Adaptive Sampling via subspace angles}

Concept of subspace angles:

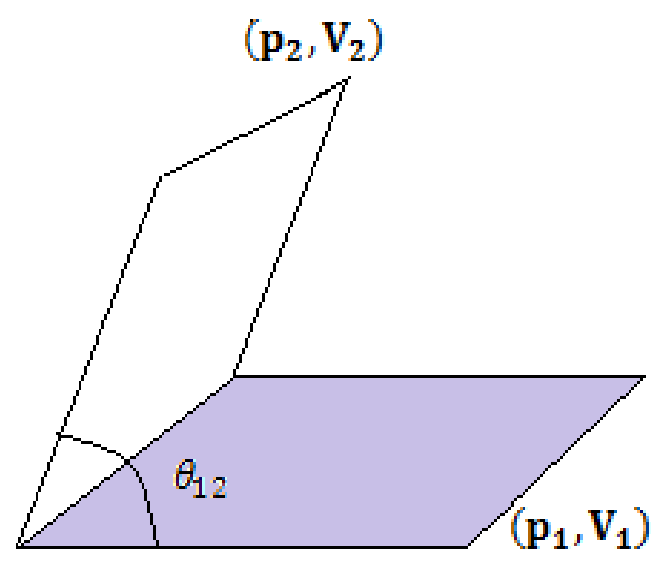

- $\mathbf{V}_{1}$ and $\mathbf{V}_{2}$ are orthonormal bases for the subspaces $\mathcal{V}_{1}$ and $\mathcal{V}_{2}$

- The largest angle between the subspaces can be determined by

$$
\begin{aligned}
& \theta_{12}=\arcsin \left(\sqrt{1-\sigma_{r}^{2}}\right)=\arccos \left(\sigma_{r}\right) \\
& \sigma_{r}: \text { smallest singular value of } \mathbf{V}_{1}^{T} \mathbf{V}_{2}
\end{aligned}
$$

Usage for adaptive grid refinement:

- The larger the subspace angle, the more different are the projection matrices, and thus:

- the higher the parametric sensitivity

- and the more sample points can be introduced in the respective sub-span

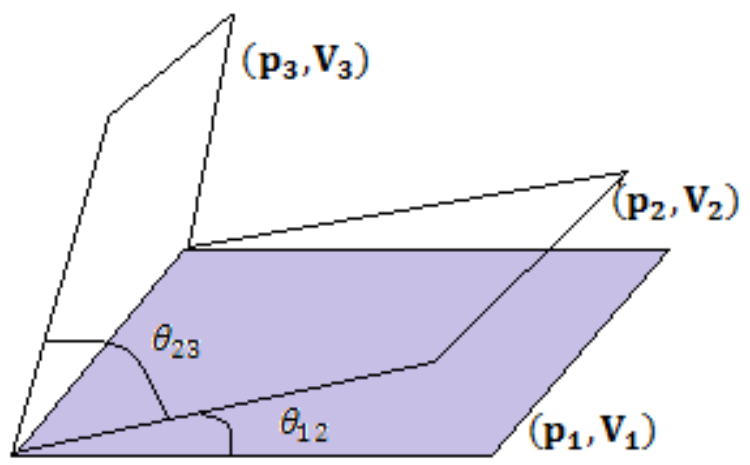




\section{Automatic Adaptive Sampling: Pseudo-Code}

1) Input $\theta_{\max }$

2) Divide the entire parameter range into a uniform grid, calling it $p_{1}, p_{2}, \cdots, p_{K}$

3) While all $l_{i, i+1}>1$ do

a) Calculate the projection matrices $\mathbf{V}_{1}, \mathbf{V}_{2}, \cdots, \mathbf{V}_{K}$ corresponding to each of these values $p_{1}, p_{2}, \cdots, p_{K}$

b) Compute subspace angles $\theta_{12}, \theta_{23}, \cdots, \theta_{K-1, K}$ between these $\mathbf{V}_{i}$ 's, each taken pairwise

c) Calculate

$$
l_{12}=\left\lceil\frac{\theta_{12}}{\theta_{\max }}\right\rceil, l_{23}=\left\lceil\frac{\theta_{23}}{\theta_{\max }}\right\rceil, \cdots, l_{K-1, K}=\left\lceil\frac{\theta_{K-1, K}}{\theta_{\max }}\right\rceil
$$

d) Divide the interval between $p_{1}$ and $p_{2}$ into $l_{12}$ further intervals. Likewise, do the same for all the other intervals.

e) Obtain new grid points $p_{1}, p_{2}, \cdots, p_{N}$, whereas $N>K$ End While
Local reduction at sample points possible using any preferred MOR technique

$$
\text { theta }(i)=
$$

subspace $(\operatorname{Vp}\{i\}, \operatorname{Vp}\{i+1\})$

Quantitative indicator of how many pieces each parameter interval is to be further broken

\section{Stopping criterion:}

1. All ratios are equal to 1

2. Specified maximum number of samples points reached

Next iteration: local reduction, etc. only at points that got added in the last while-loop iteration (efficient!) 


\section{Numerical example: Timoshenko Beam}

- Finite element 3D model of a Timoshenko beam

- Parameter is the length of the beam: $p \equiv L$

- One-sided Krylov reduction with shifts at $s_{0}=0$

- $\theta_{\max }=10^{\circ}$ chosen

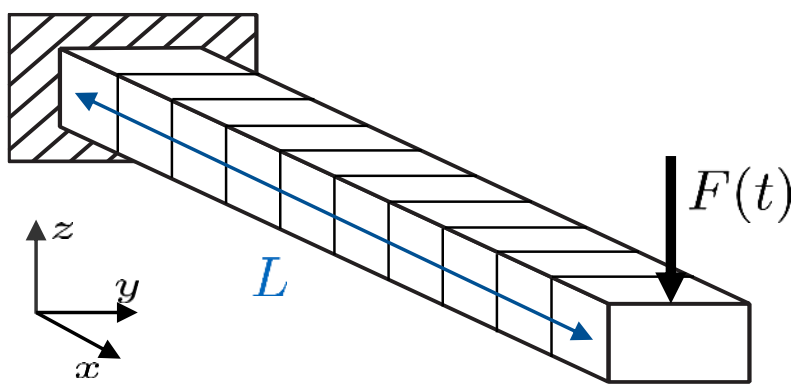

Table 1: Sample points $p_{i}$, subspace angles $\theta_{i, i+1}$ and ratios $l_{i, i+1}$

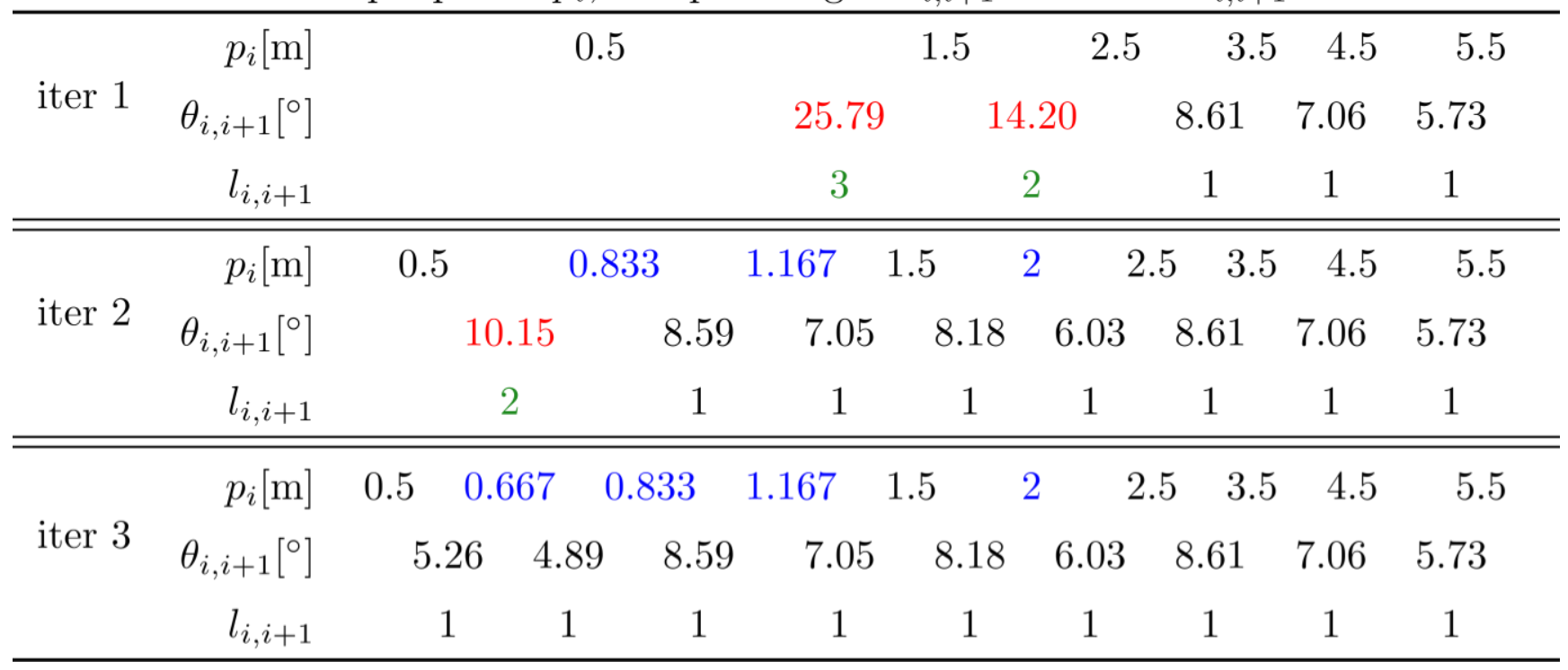




\section{Numerical example: Timoshenko Beam}

Initial uniform grid with $K=6$

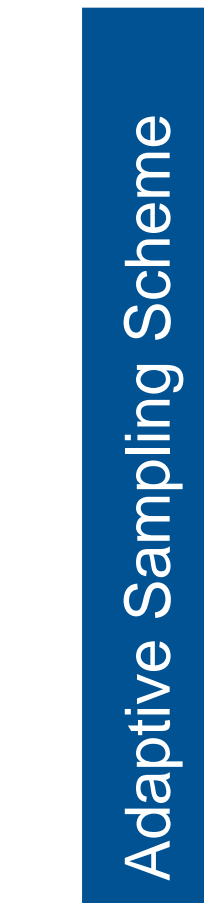

Table 1: Sample points $p_{i}$, subspace angles $\theta_{i, i+1}$ and ratios $l_{i, i+1}$

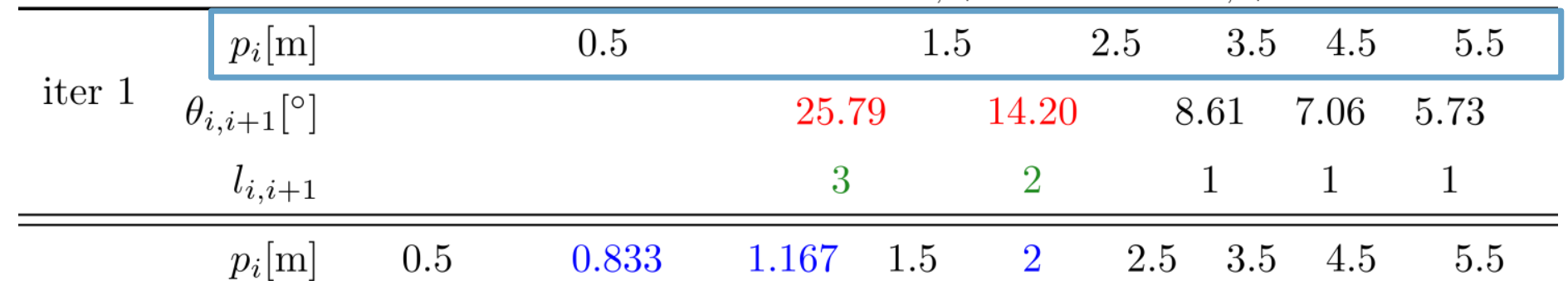

$\begin{array}{llllllllll}\text { iter } 2 & \theta_{i, i+1}\left[^{\circ}\right] & 10.15 & 8.59 & 7.05 & 8.18 & 6.03 & 8.61 & 7.06 & 5.73\end{array}$

\begin{tabular}{rrrrrrrrrrrrrr} 
& $l_{i, i+1}$ & & 2 & & 1 & 1 & 1 & 1 & 1 & 1 & 1 \\
\hline \hline \multirow{2}{*}{ iter 3 } & $p_{i}[\mathrm{~m}]$ & 0.5 & 0.667 & 0.833 & 1.167 & 1.5 & 2 & 2.5 & 3.5 & 4.5 & 5.5 \\
& $\theta_{i, i+1}\left[^{\circ}\right]$ & 5.26 & 4.89 & 8.59 & 7.05 & 8.18 & 6.03 & 8.61 & 7.06 & 5.73
\end{tabular}

\begin{tabular}{llllll}
$l_{i, i+1}$ & 1 & 1 & 1 & \\
\hline & & \\
\hline
\end{tabular}

Final refined grid with $N=10$

Interpolation point $p^{\text {int }}=1.0$

between ROM 3 \& ROM 4 


\section{Numerical results - Direct vs. Interpolated ROM}

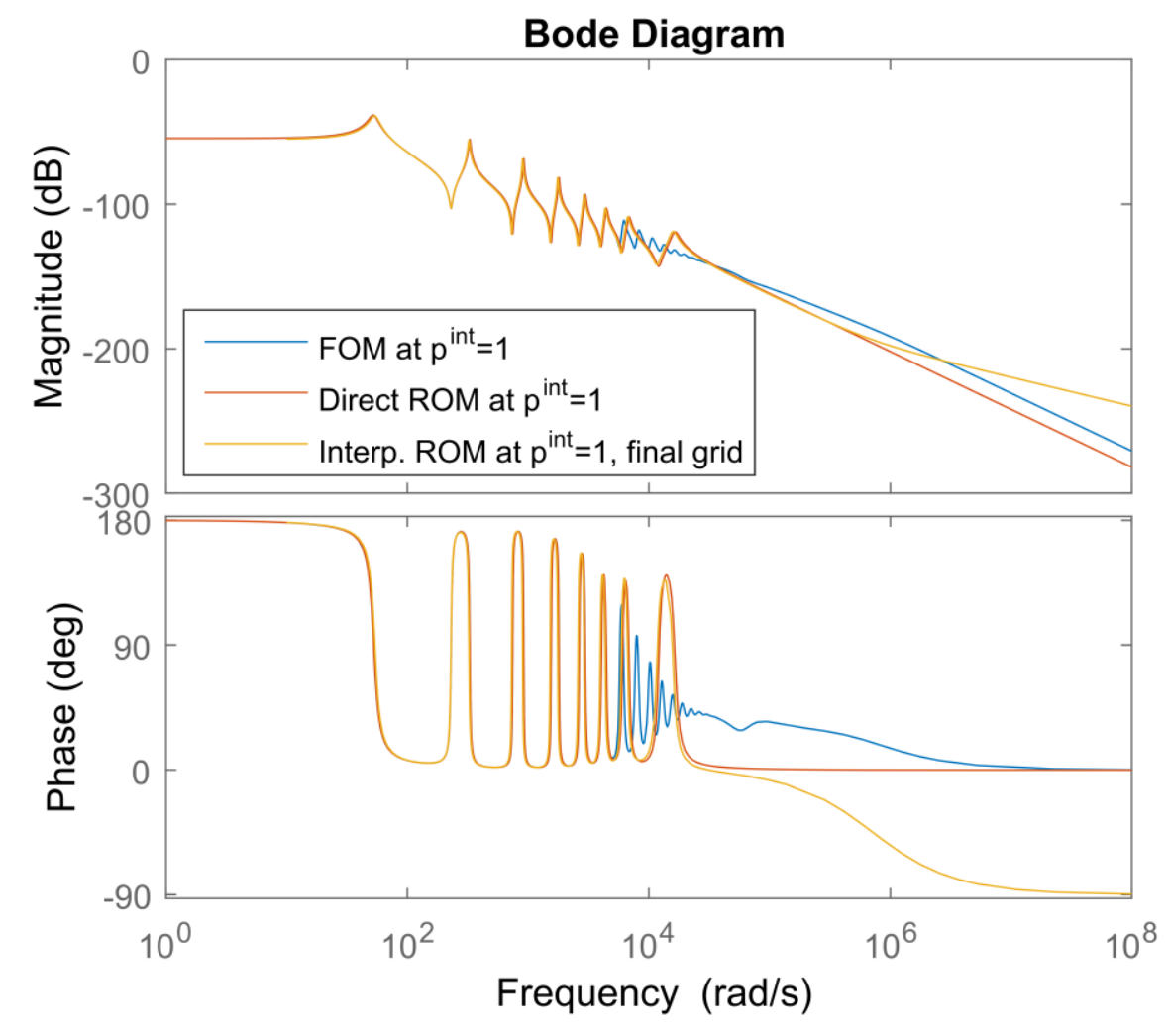

FOM size $n=240$, ROMs size $r=17$

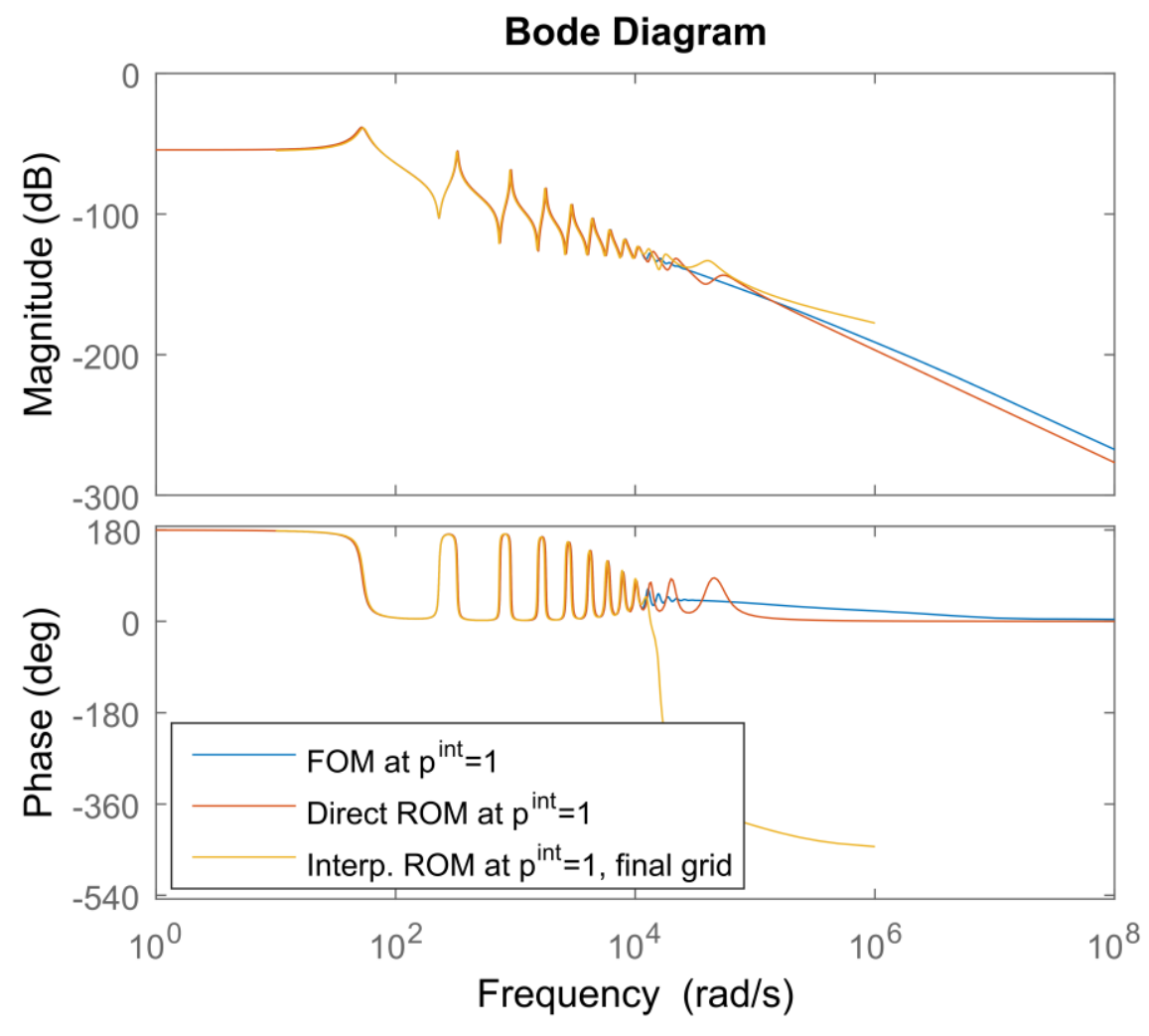

FOM size $n=2400$, ROMs size $r=25$

Two errors: model reduction error + interpolation error 


\section{Numerical results - Direct vs. Interpolated ROM}

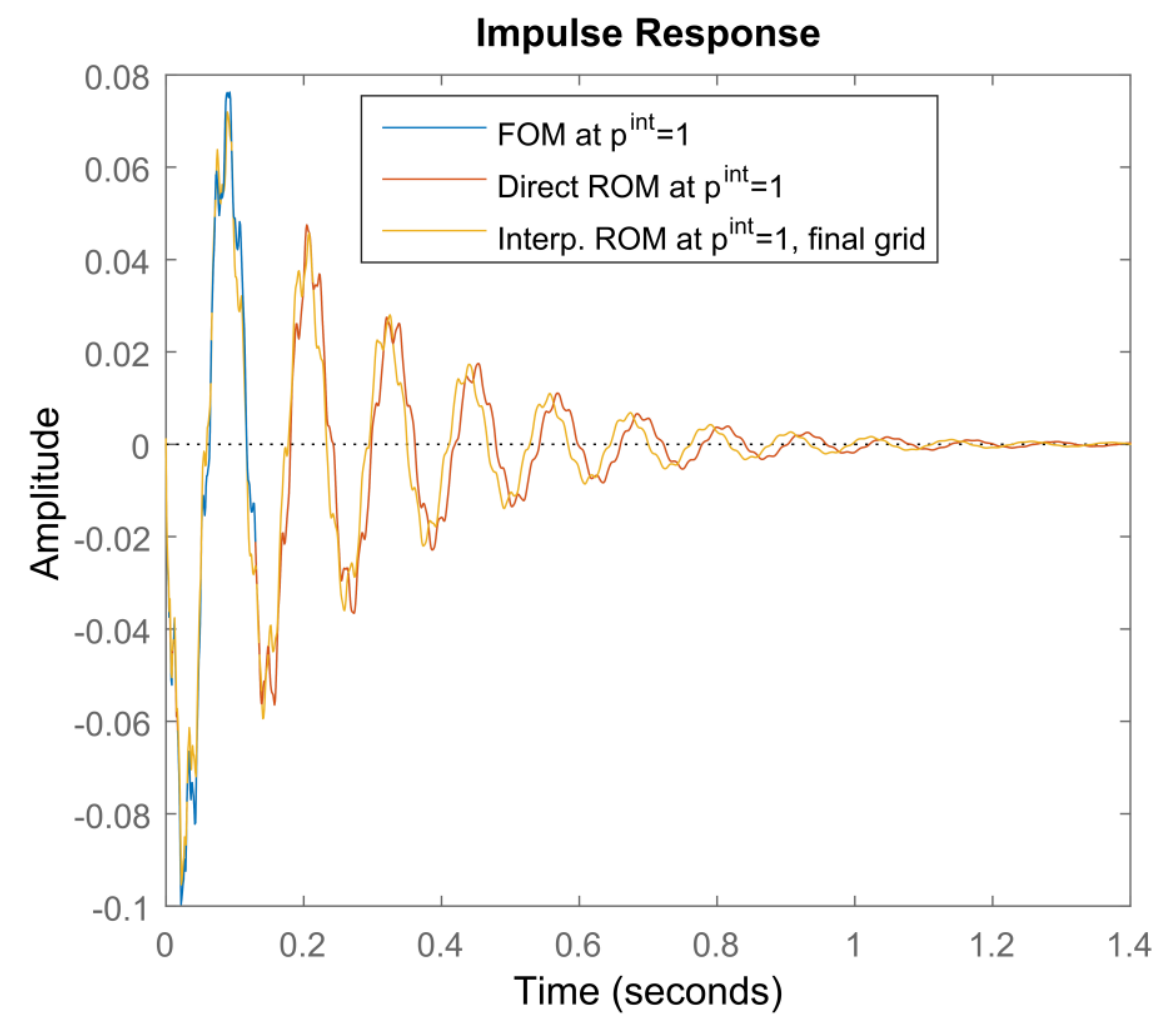

FOM size $n=2400$, ROMs size $r=25$

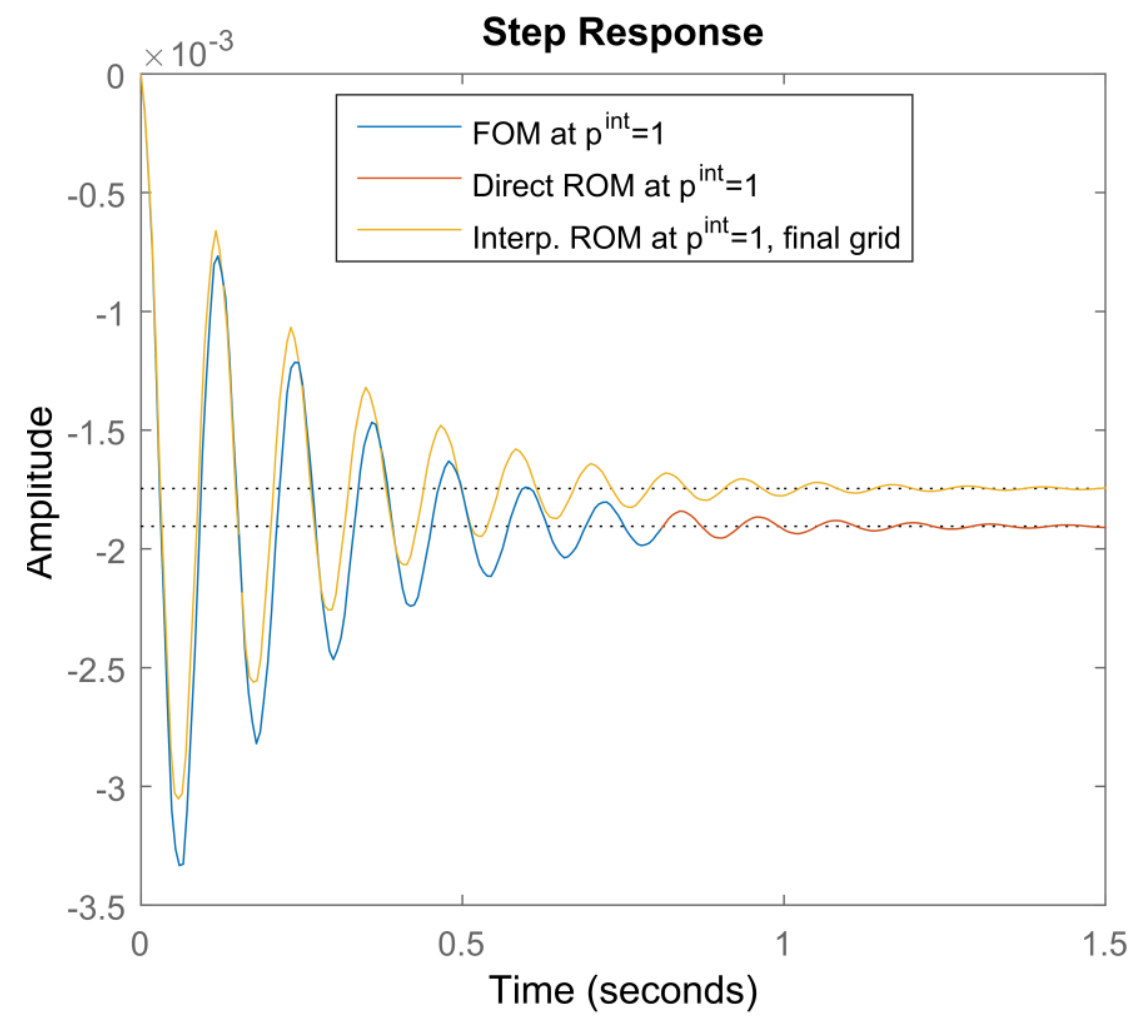

FOM size $\mathrm{n}=2400$, ROMs size $r=25$

With MatrInterp: no need to reduce the model for every new parameter value 


\section{Numerical results - Initial vs. Final Grid}

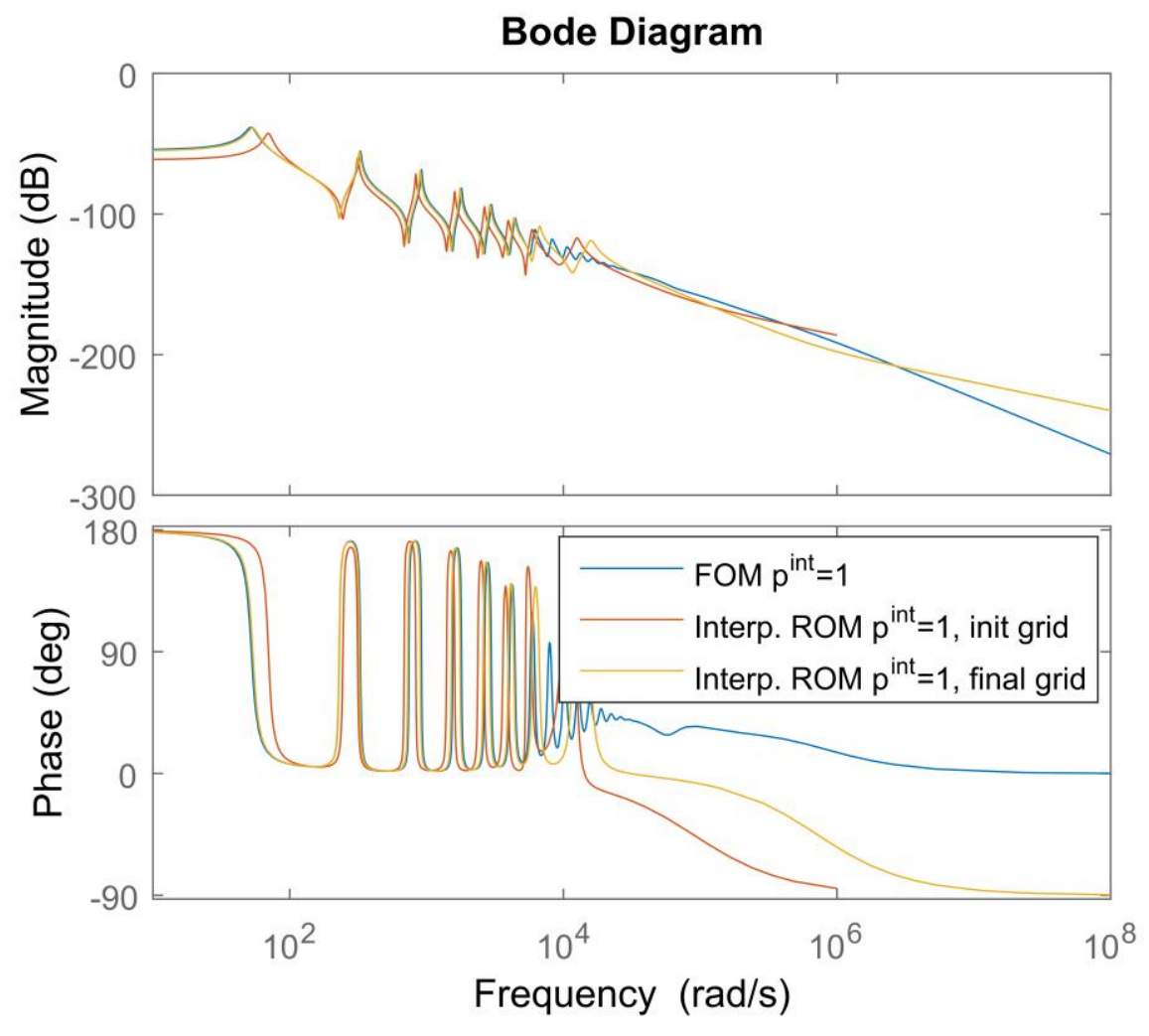

FOM size $n=240$, ROMs size $r=17$

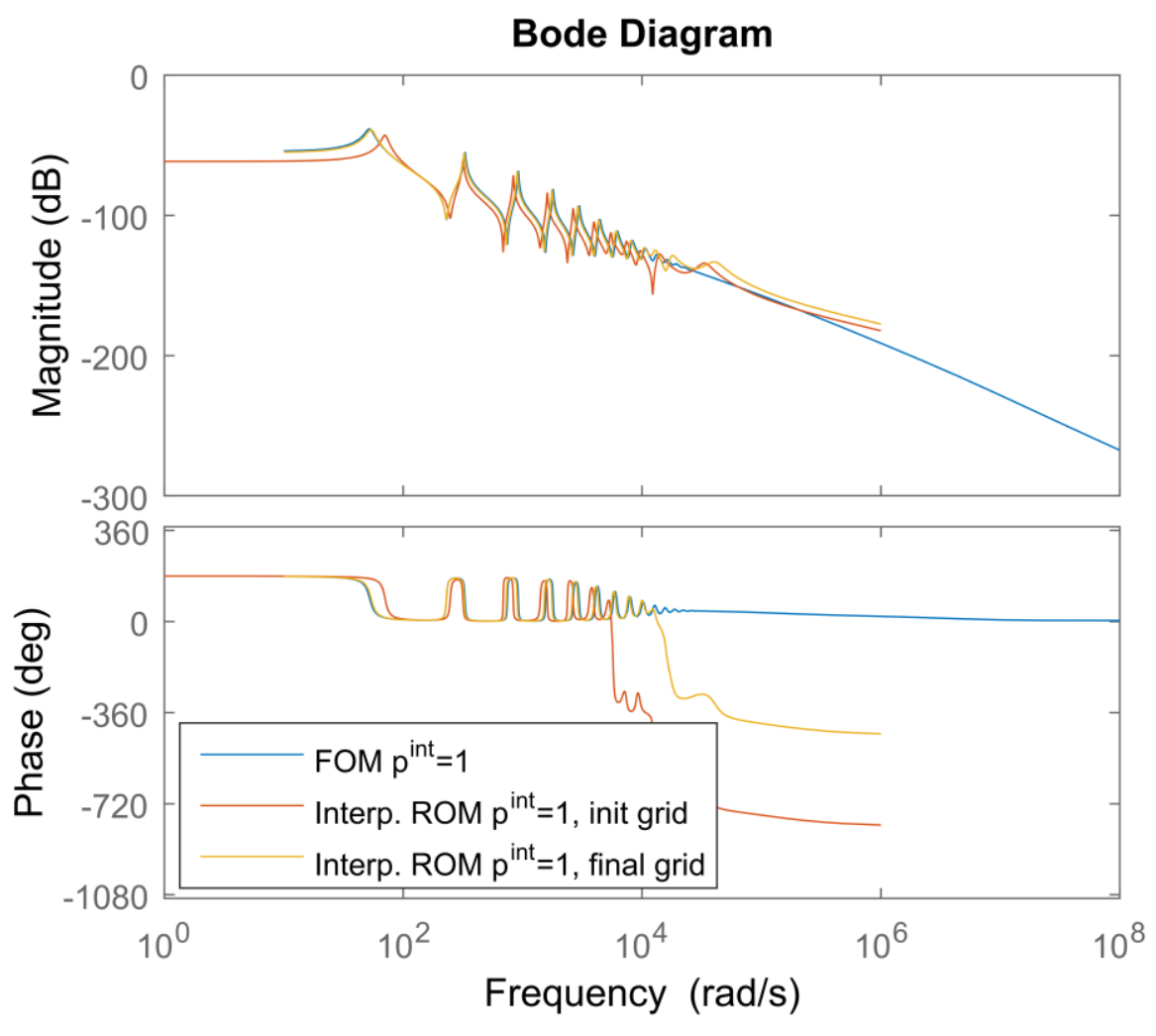

FOM size $n=2400$, ROMs size $r=25$

ROMs calculated with the final grid yield better approximations 


\section{Numerical results - Initial vs. Final Grid}

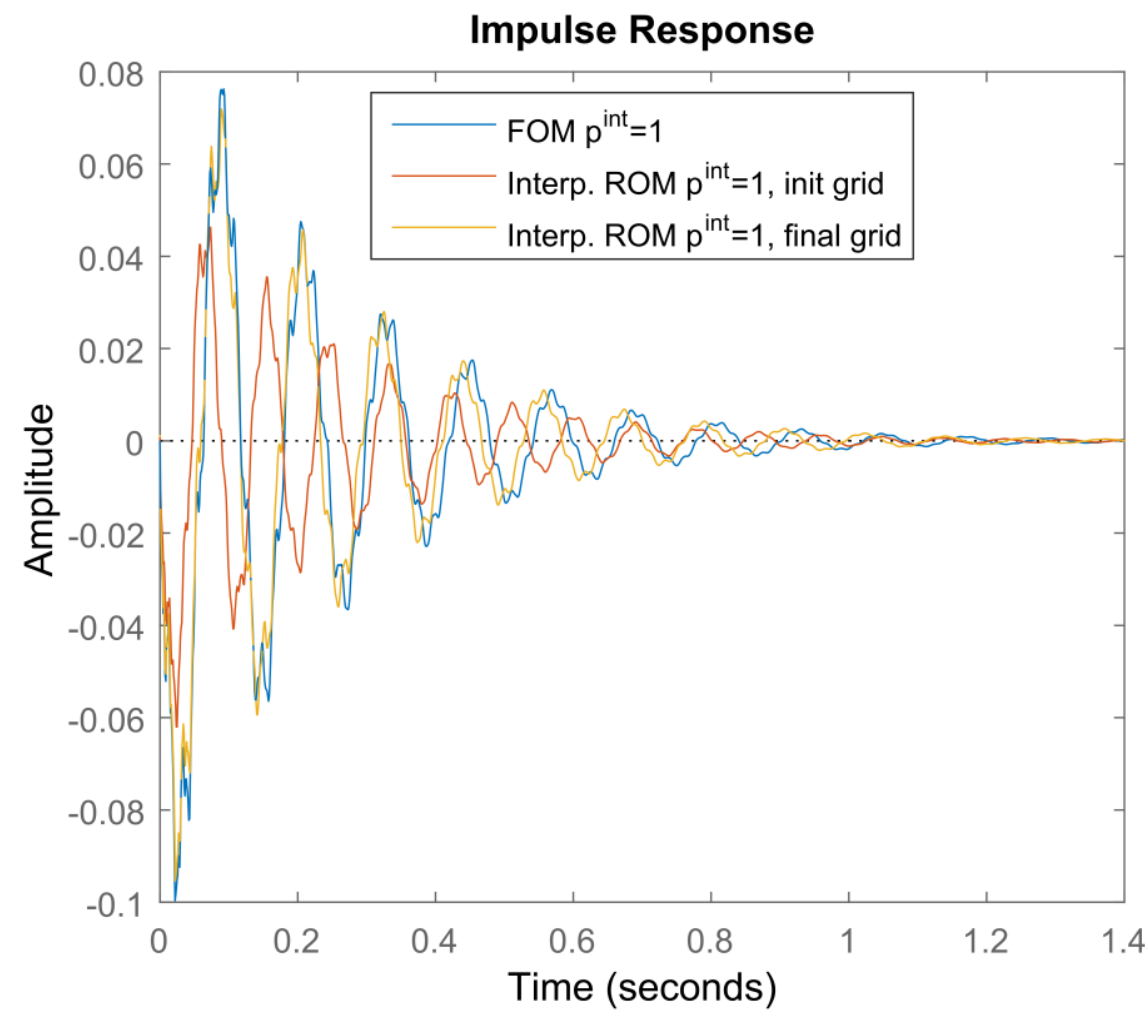

FOM size $\mathrm{n}=2400$, ROMs size $\mathrm{r}=25$

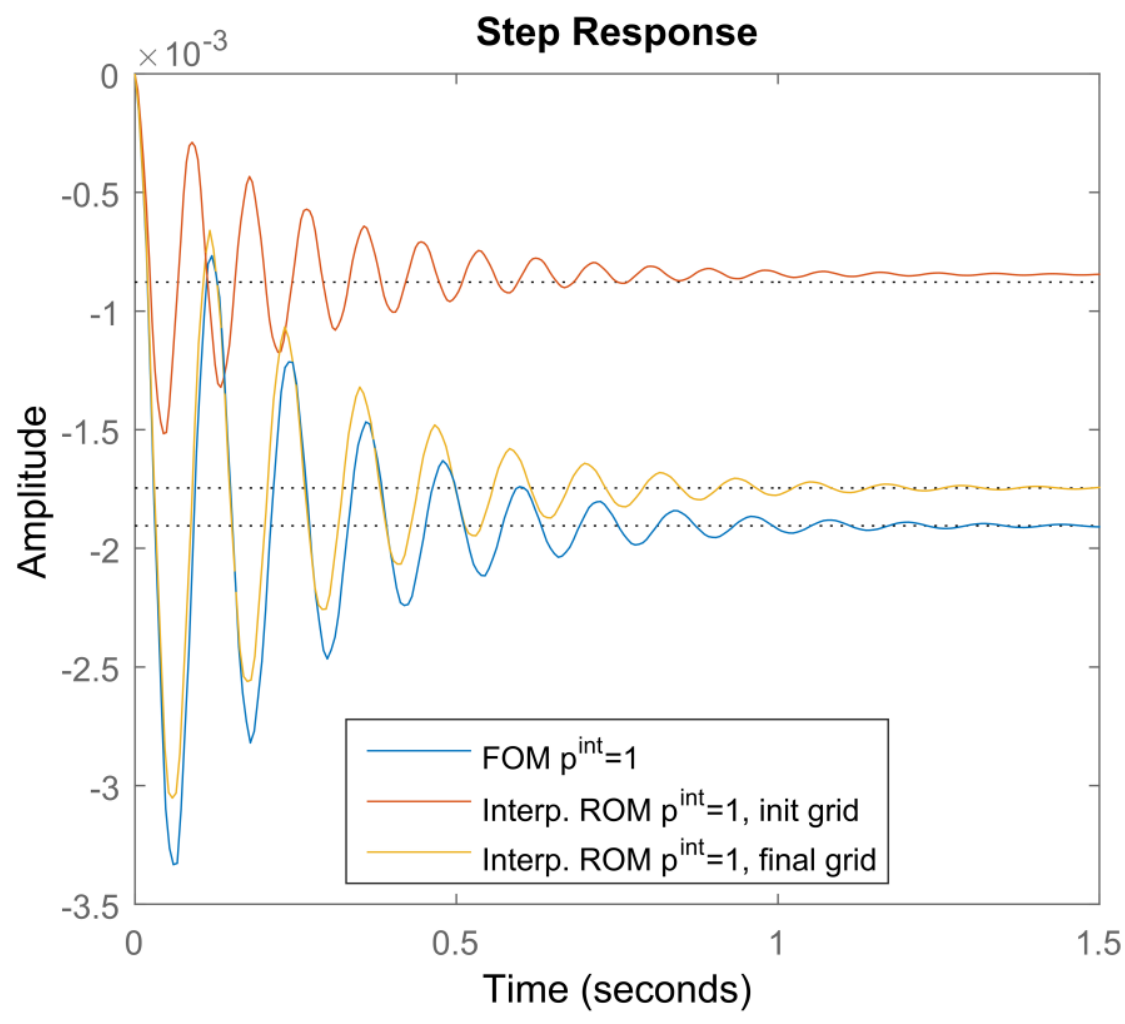

FOM size $n=2400$, ROMs size $r=25$

ROMs calculated with the final grid yield better approximations 


\section{Numerical results - Initial vs. Final Grid}

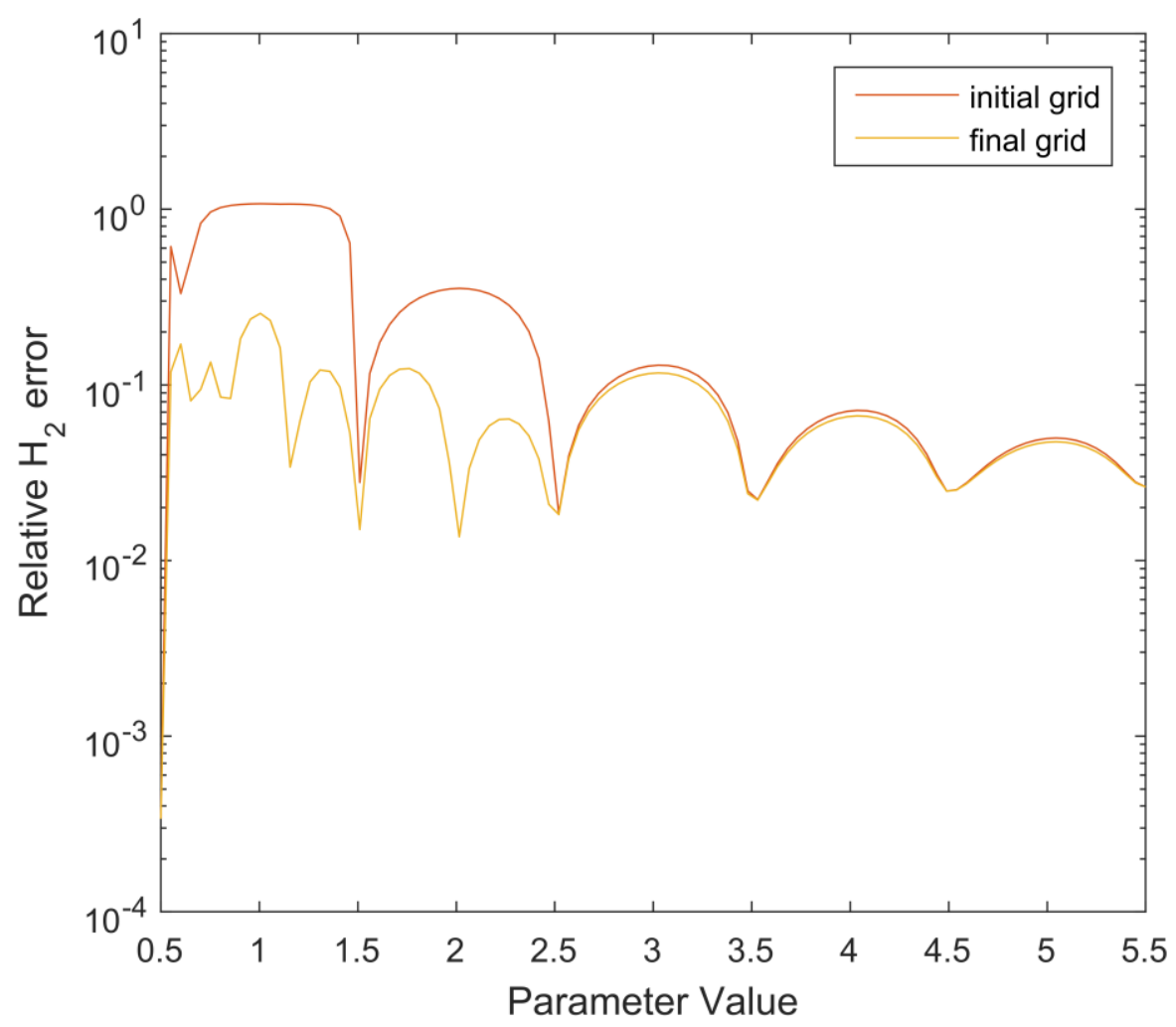

- Quantitative evaluation of the approximation

- Relative $\mathrm{H} 2$ error for $\mathrm{n} \mathrm{P}=100$ different query points $p^{\text {int }}$

- Errors particularly small in the proximity of the sample points

- Final grid yields smaller errors for smaller beam lengths due to the adaptive refinement in this region

Relative $\mathrm{H} 2$ error between FOMs and interpolated ROMs for different parameter values and grids: FOM size $n=240, R O M$ size $r=17$ 


\section{ps ssMOR Toolbox - Analysis and Reduction of}

\section{Parametric Models in}

$\checkmark$ Definition of parametric sparse statespace models

psys $=$ loadFemBeam3D (Opts)
psys $=$ loadAnemometer3parameter

$\checkmark$ Manipulation of psss-class objects

psys = fixparameter (psys, $2,1.7)$

psys $=$ unfixparameter (psys, 3$)$

$\checkmark$ Compatible with the sss \& sssMOR toolboxes

param $=[\mathrm{p} 1, \mathrm{p} 2, \mathrm{p} 3, \mathrm{p} 4]$

sys $=$ psys (param)

bode (psys, param); step (sys); $\checkmark$ Different parametric reduction methods available (offline- \& online-phase)

$$
\begin{aligned}
& \text { psysr = matrInterpoffline } \\
& \text { (psys, param,r, opts); } \\
& \text { psysr = globalPmoroffline } \\
& \text { (psys, param,r, opts) } \\
& \text { sysr = psysr(pInterp) }
\end{aligned}
$$

localReduction \& adaptiveSampling as core functions

Coming SoOn! www.rt.mw.tum.de/?morlab 


\section{Summary \& Outlook}

\section{Takehome Messages:}

- A simple automatic sampling strategy is presented for adaptively choosing sample points in parametric model order reduction

- Scheme uses concept of subspace angles to measure need of further sampling points

- Adaptive approach is fully automated and embedded in the matrix interpolation framework

- Algorithm is applied to a Timoshenko beam model, achieving satisfactory results.

Future Extensions / Ongoing Work:

- Extension of proposed adaptive sampling scheme to 2D and 3D parametric case

- Higher dimensional case $(d>3)$ with adaptive sparse grids is topic of future research

- psssMOR toolbox is being actively developed and will be available open-source very soon!!

\section{Thank you for your attention!}


Backup 


\section{References}

[Amsallem '10]

[Bazaz et al. '15]

[Benner et al. '15]

[Baur et al. '15]

[Geuss et al. '08]

[Panzer et al. '10]
Interpolation on manifolds of CFD-based fluid and finite elementbased structural reduced-order models for on-line....

Adaptive Parameter Space Sampling in Matrix Interpolatory pMOR.

A survey of projection-based model reduction methods for parametric dynamical systems.

Comparison of methods for parametric model order reduction of instationary problems.

On Parametric Model Order Reduction by Matrix Interpolation.

Parametric Model Order Reduction by Matrix Interpolation. 


\section{Numerical results - Direct vs. Interpolated ROM}

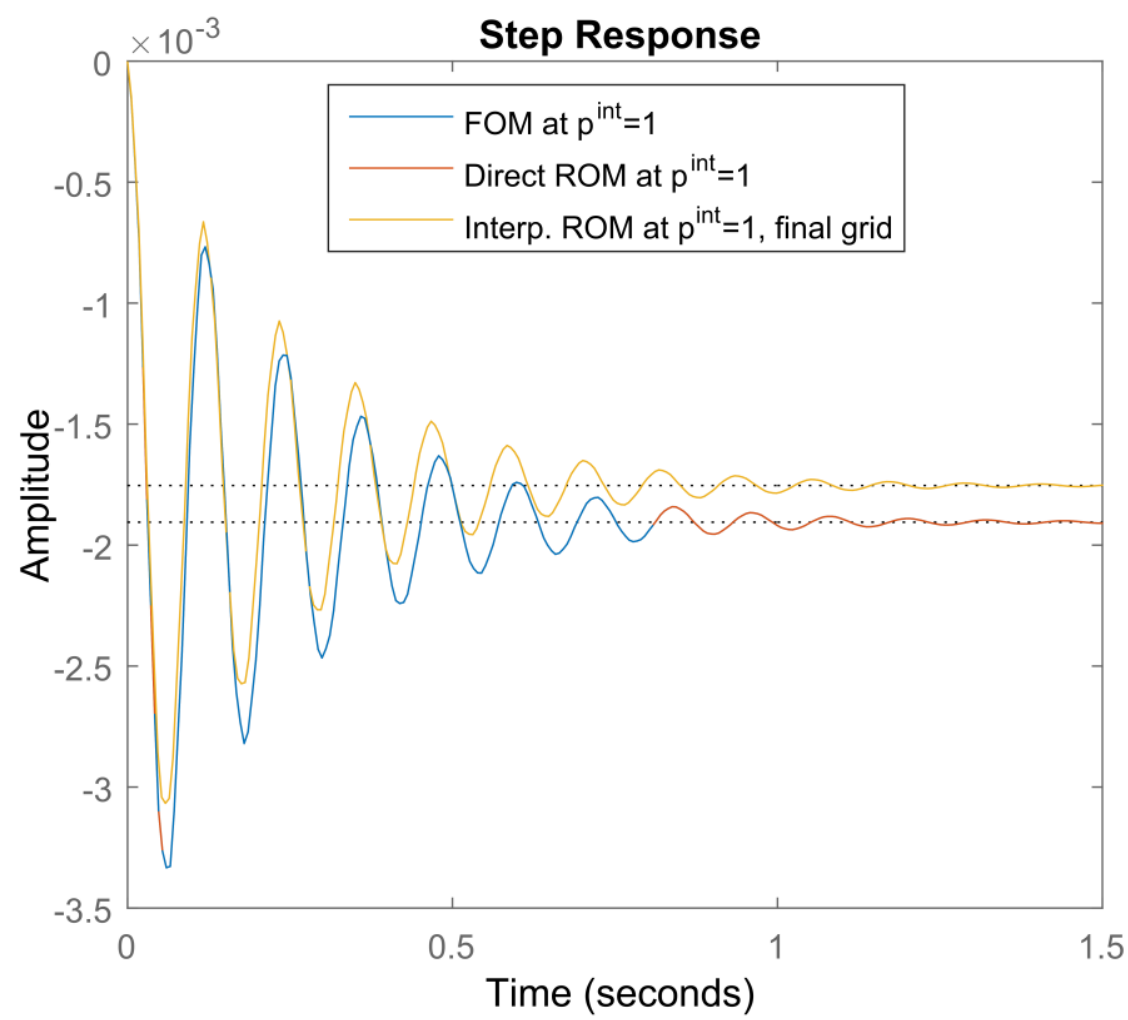

FOM size $n=240$, ROMs size $r=17$

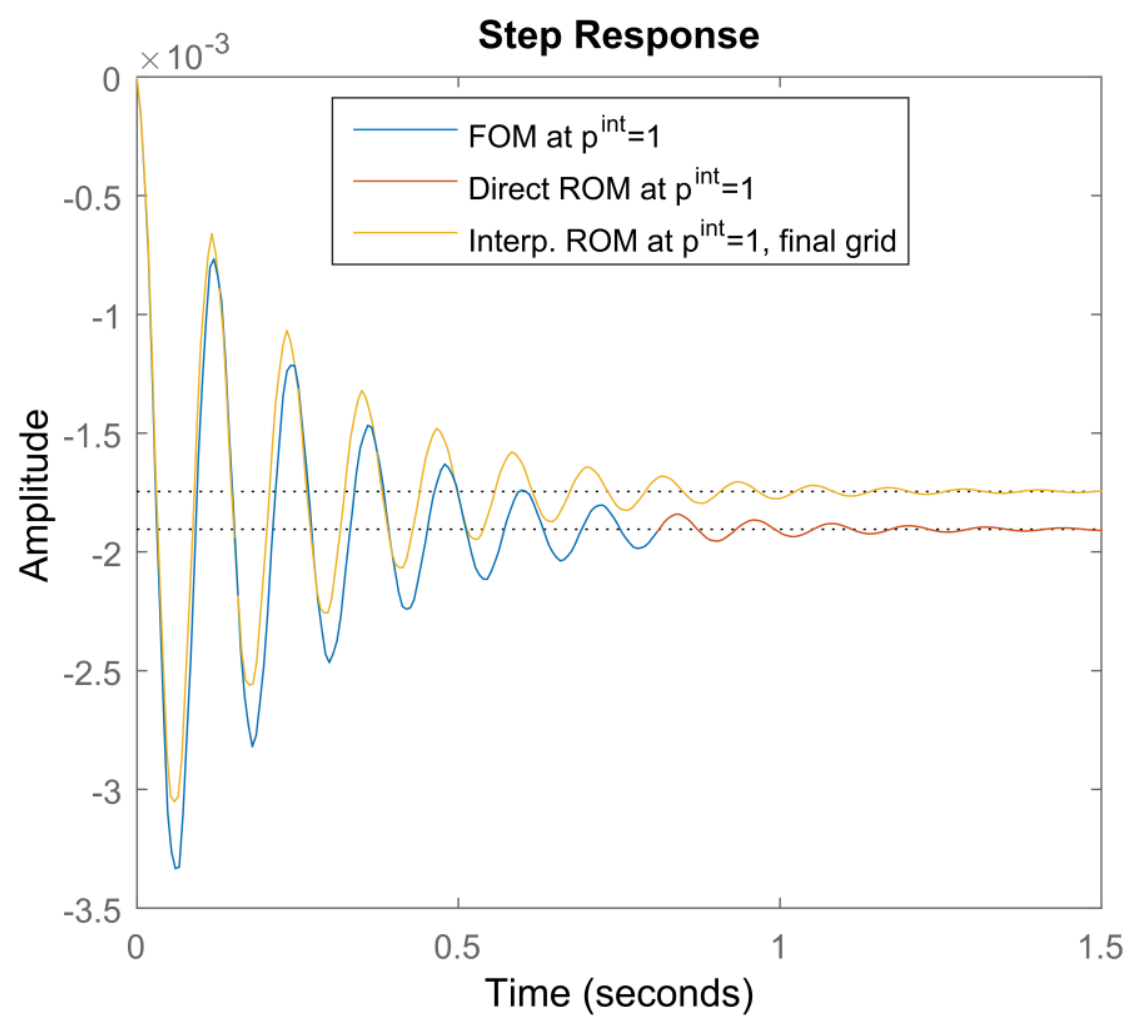

FOM size $n=2400$, ROMs size $r=25$ 


\section{Numerical results - Direct vs. Interpolated ROM}

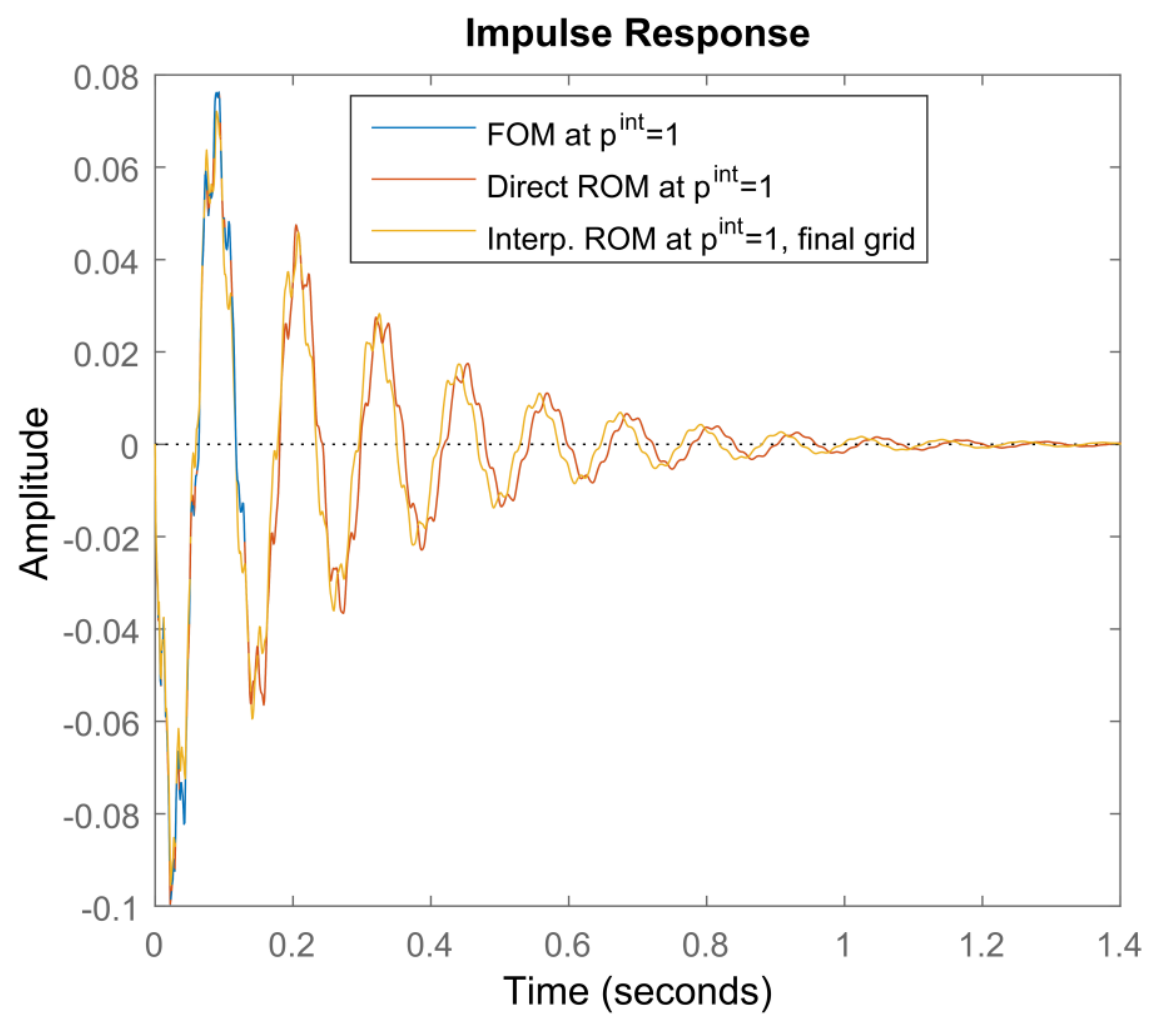

FOM size $n=240$, ROMs size $r=17$

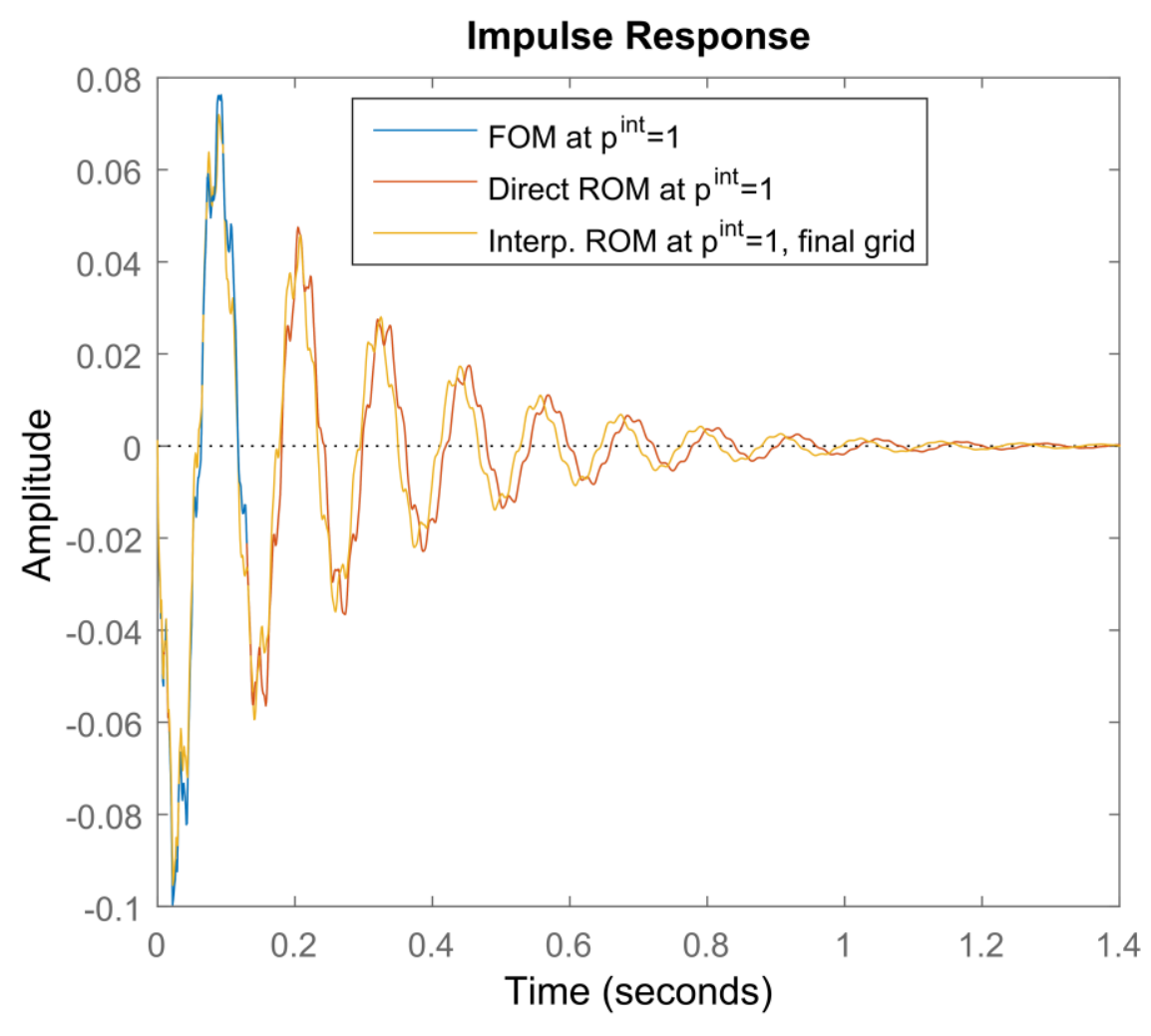

FOM size $n=2400$, ROMs size $r=25$ 


\section{Numerical results - Initial vs. Final Grid}

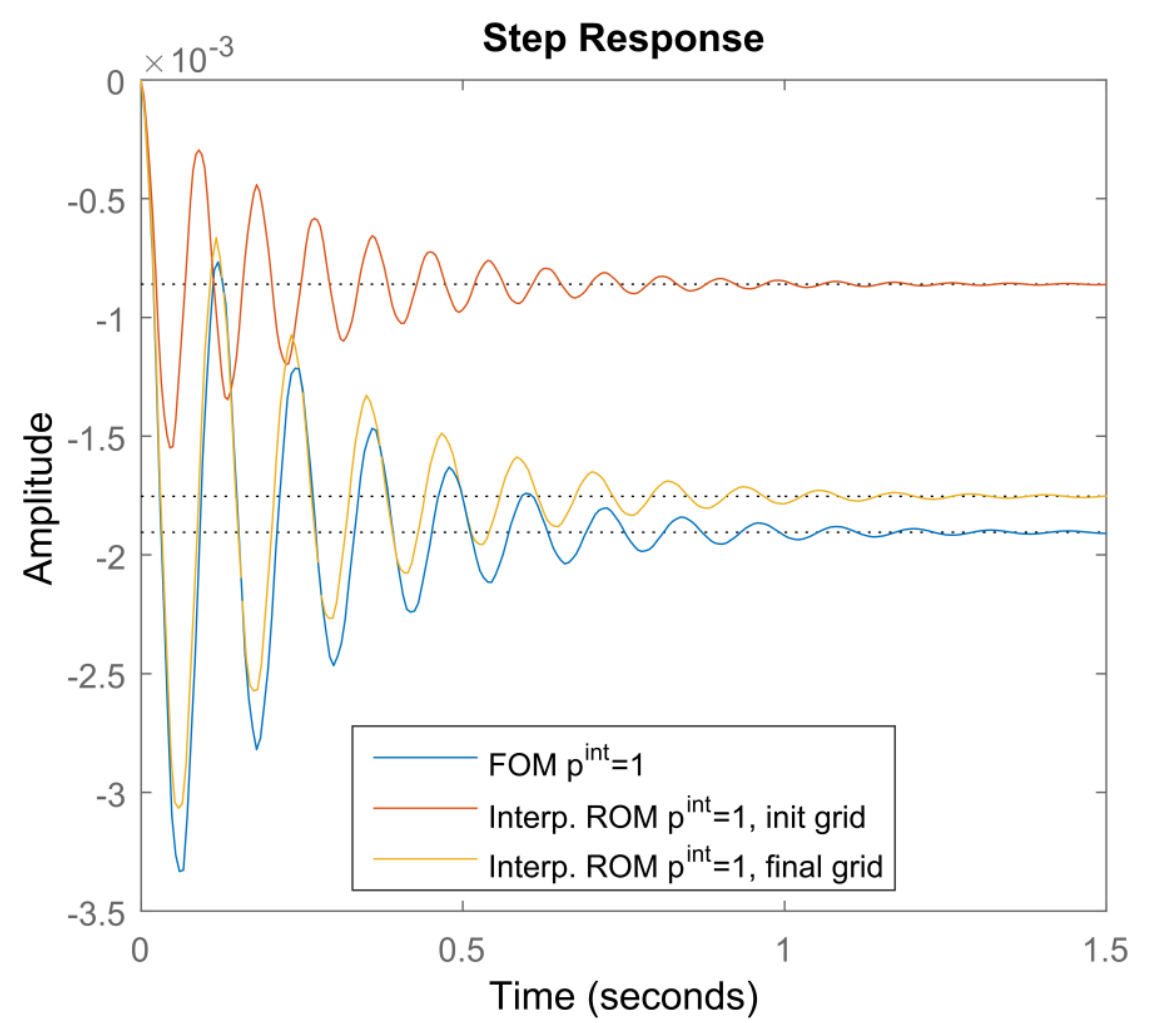

FOM size $n=240$, ROMs size $r=17$

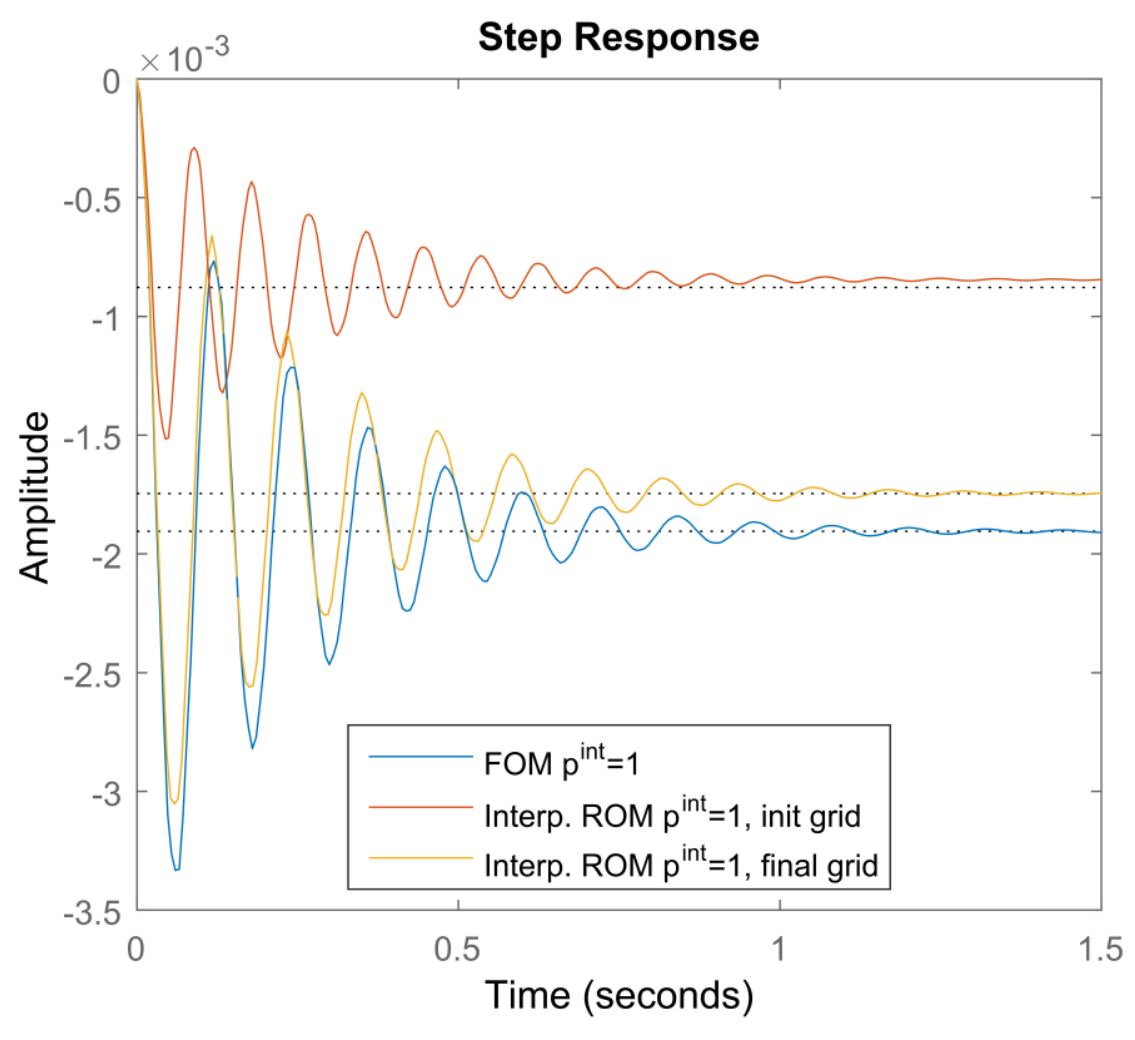

FOM size $n=2400$, ROMs size $r=25$ 


\section{Numerical results - Initial vs. Final Grid}

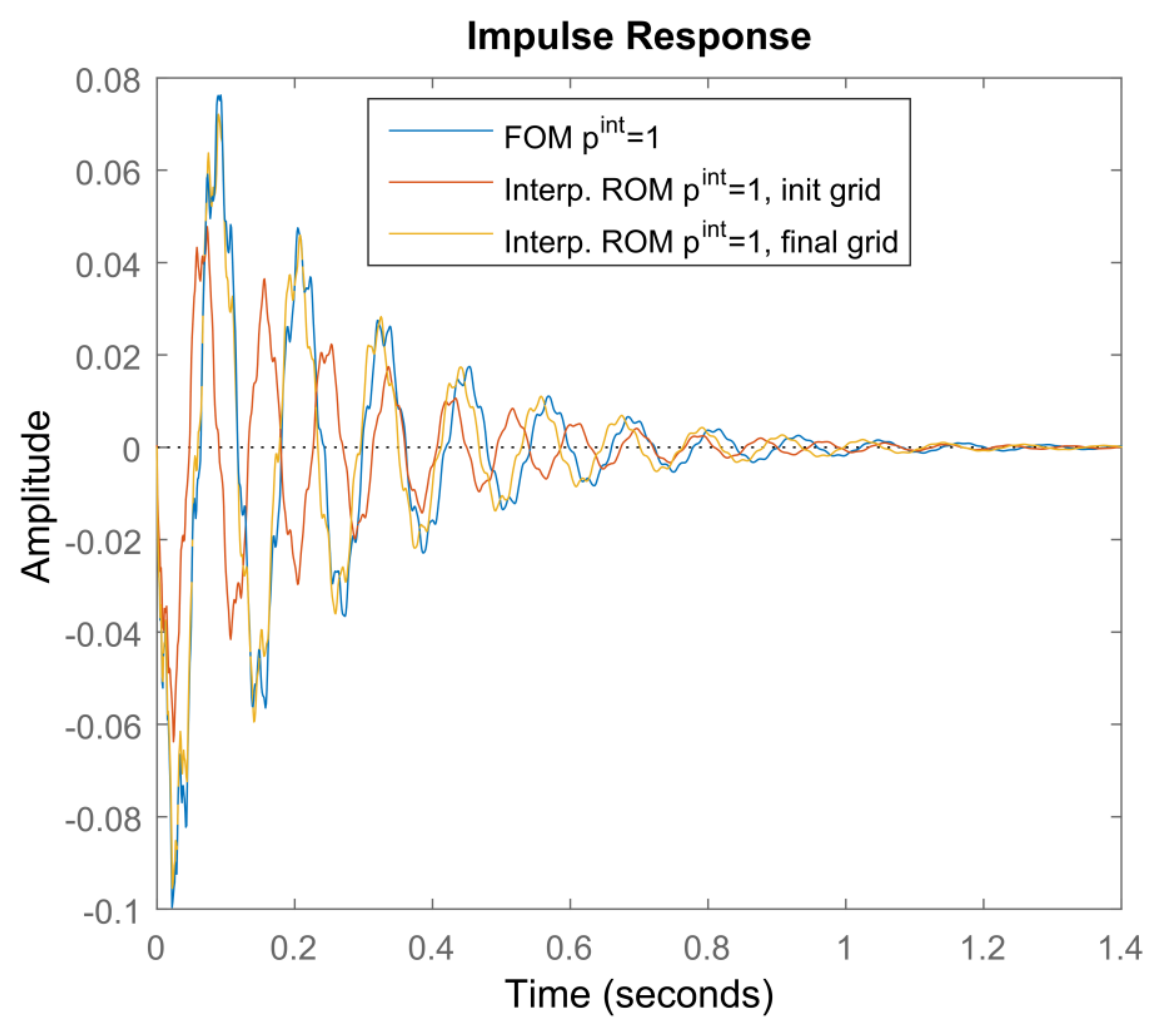

FOM size $n=240$, ROMs size $r=17$

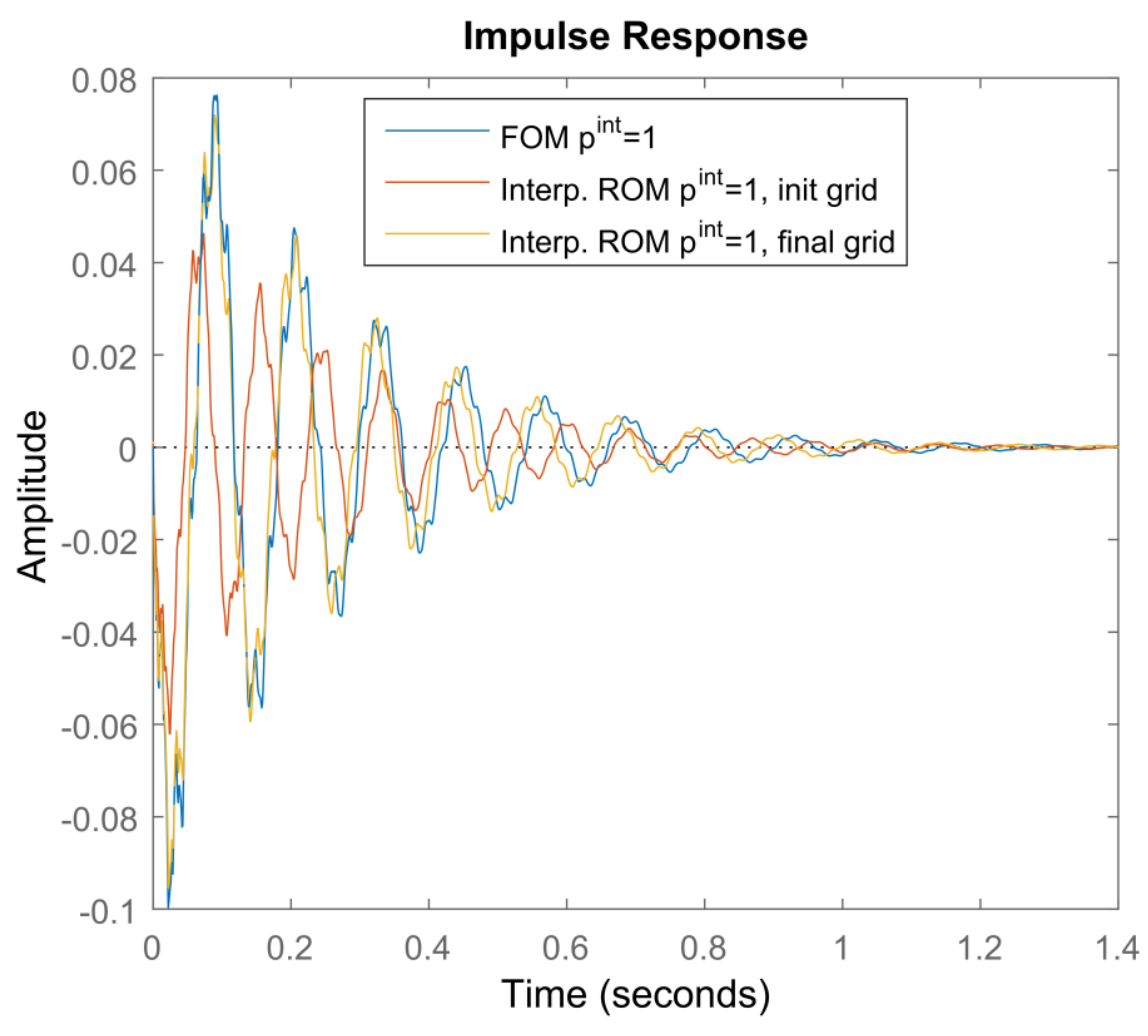

FOM size $n=2400$, ROMs size $r=25$ 
sss \& sssMOR - MATLAB Toolboxes
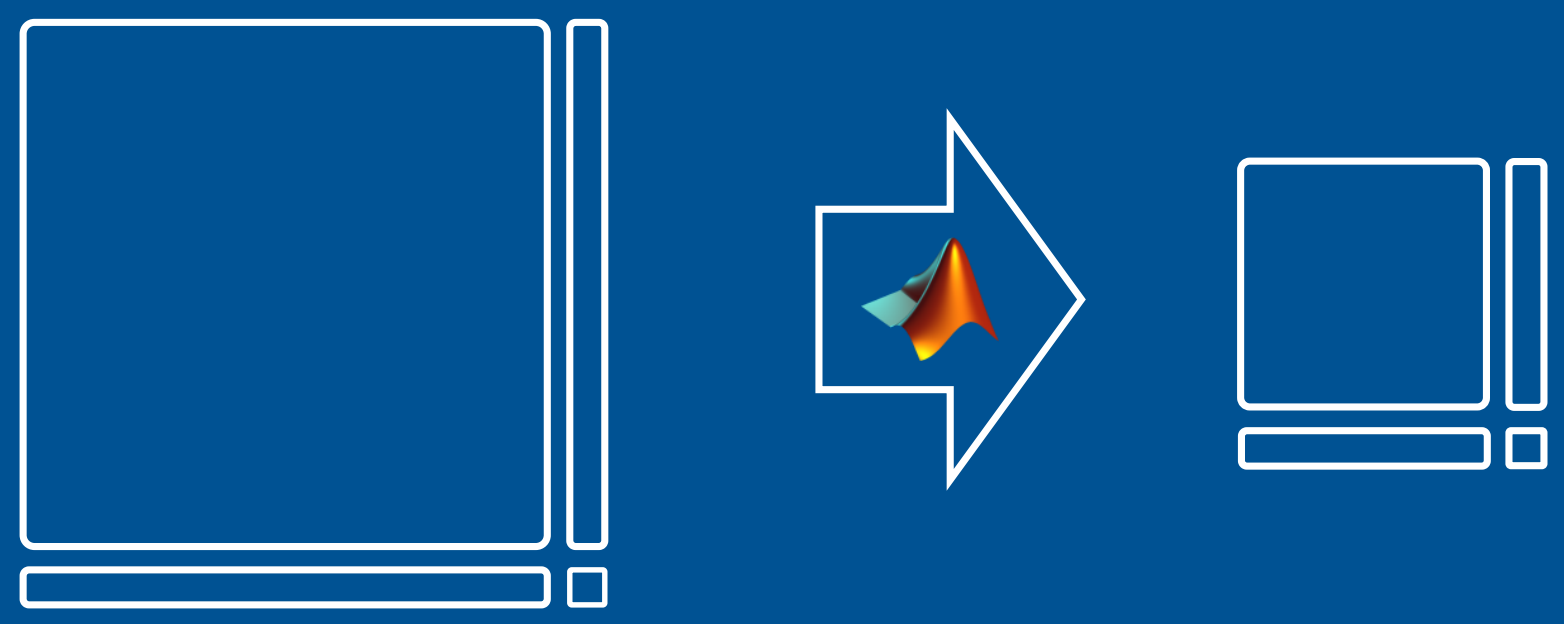


\section{Toolboxes for sparse, large-scale models in}

S SS

$$
\text { sys }=\operatorname{sss}(A, B, C, D, E) \text {; }
$$
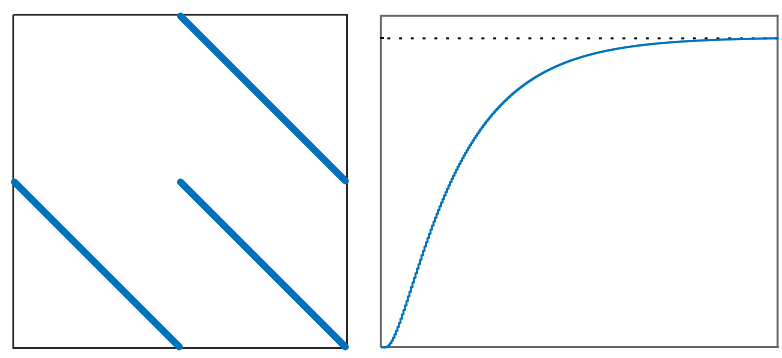

bode (sys), sigma (sys)

step (sys), impulse (sys)

norm (sys, 2), norm (sys, inf)

c2d, lsim, eigs, connect,...

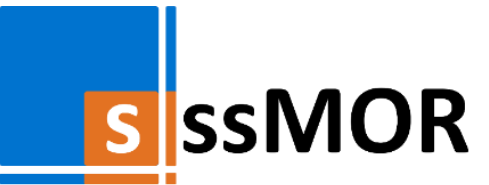

$$
\begin{aligned}
& \text { sysr }=\operatorname{tbr}(\text { sys,r) } \\
& \text { sysr }=\operatorname{rk}(\text { sys, s0) } \\
& \text { sysr }=\operatorname{irka}(\text { sys, s0) } \\
& \text { sysr }=\operatorname{cure}(\text { sys }) \\
& \text { sysr }=\operatorname{cirka}(\text { sys, s0) }
\end{aligned}
$$
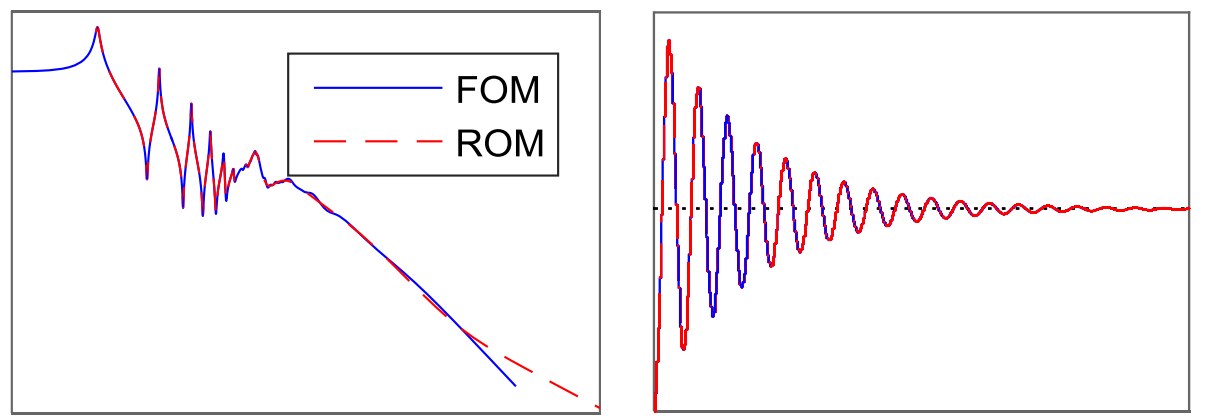

Powered by: M-M.E.S.S. toolbox [Saak, Köhler, Benner] for Lyapunov equations Available at www.rt.mw.tum.de/?sssMOR [Castagnotto/Cruz Varona/Jeschek/Lohmann '17]: ,sss \& sssMOR: Analysis and Reduction of Large-Scale Dynamic Systems in MATLAB“, at-Automatisierungstechnik] 


\section{Main characteristics}

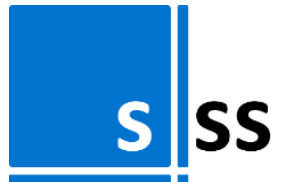

$\checkmark$ State-space models of very high order on a standard computer $\mathcal{O}\left(10^{8}\right)$

$\checkmark$ Many Control System Toolbox functions, revisited to exploit sparsity

$\checkmark$ Allows system analysis in frequency (bode, sigma,...) and time domain (step, norm, lsim,...), as well as manipulations

(connect, truncate, ...)

$\checkmark$ Is compatible with the built-in syntax

$\checkmark$ New functionality: eigs, residue, pzmap , ...

\section{SSMOR}

$\checkmark$ Classical (modalMor, tbr, rk,...) and state-of-the-art (isrk, irka, cirka, cure,...) reduction methods

$\checkmark$ Both highly-automatized

$$
\text { sysr }=\operatorname{irka}(\text { sys, n) }
$$

and highly-customizable

$$
\begin{aligned}
& \text { Opts.maxiter }=100 \\
& \text { Opts.tol = } 1 e-6 \\
& \text { Opts.stopcrit = 'combAll' } \\
& \text { Opts.verbose = true } \\
& \text { sysr = irka (sys, n, Opts) }
\end{aligned}
$$

solvelse and lyapchol as core functions 


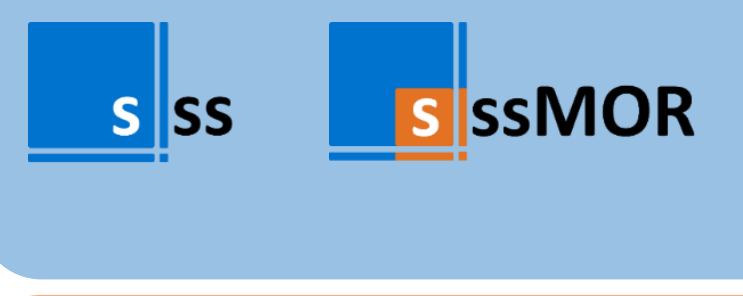

Comprehensive documentation with examples and references

\section{sssMOR App} graphical user interface

completely free and open source (contributions welcome)

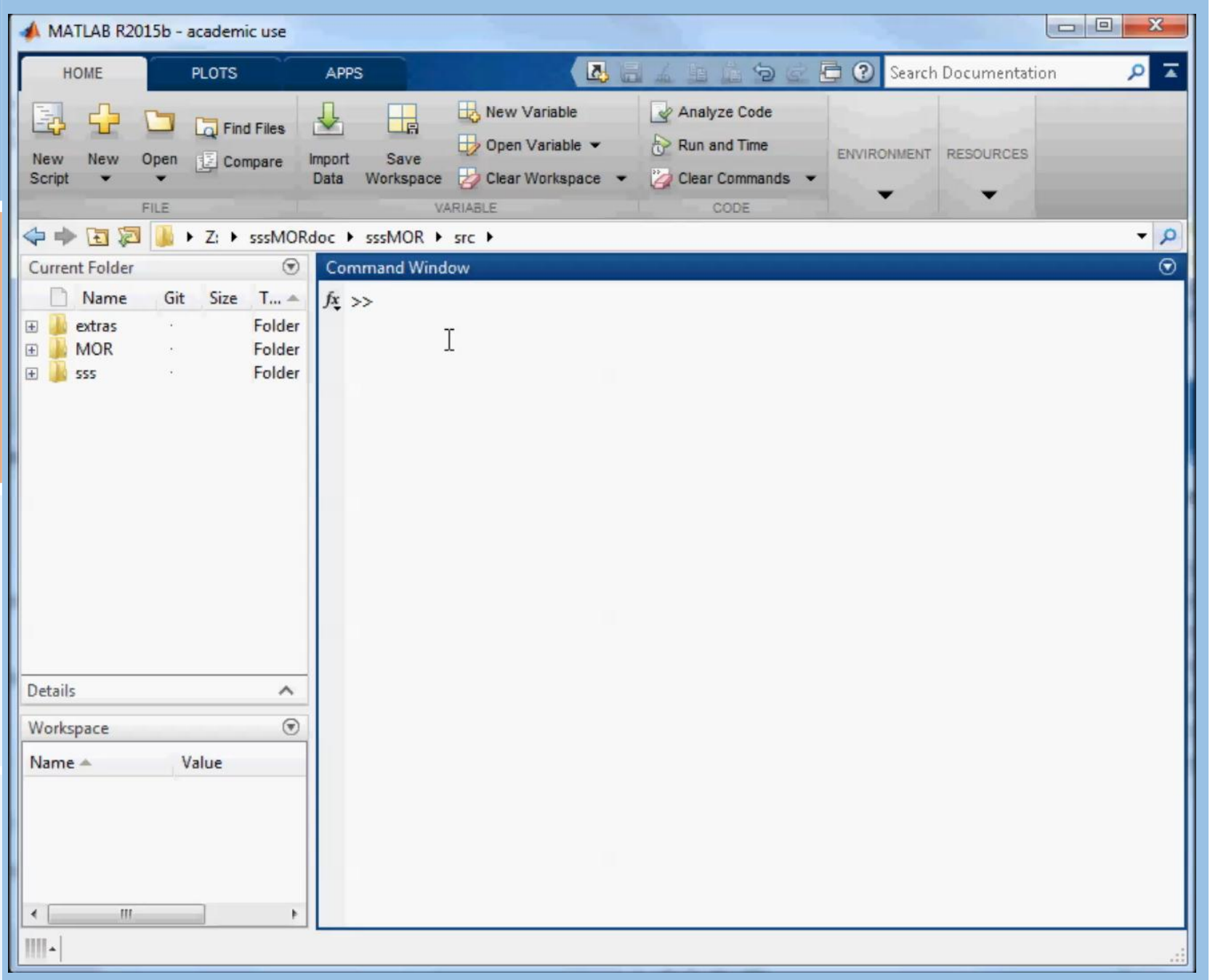




\section{$s \mid s s \quad$ s IssMOR}

Comprehensive documentation with examples and references

\section{sssMOR App} graphical user interface

completely free and open source (contributions welcome)

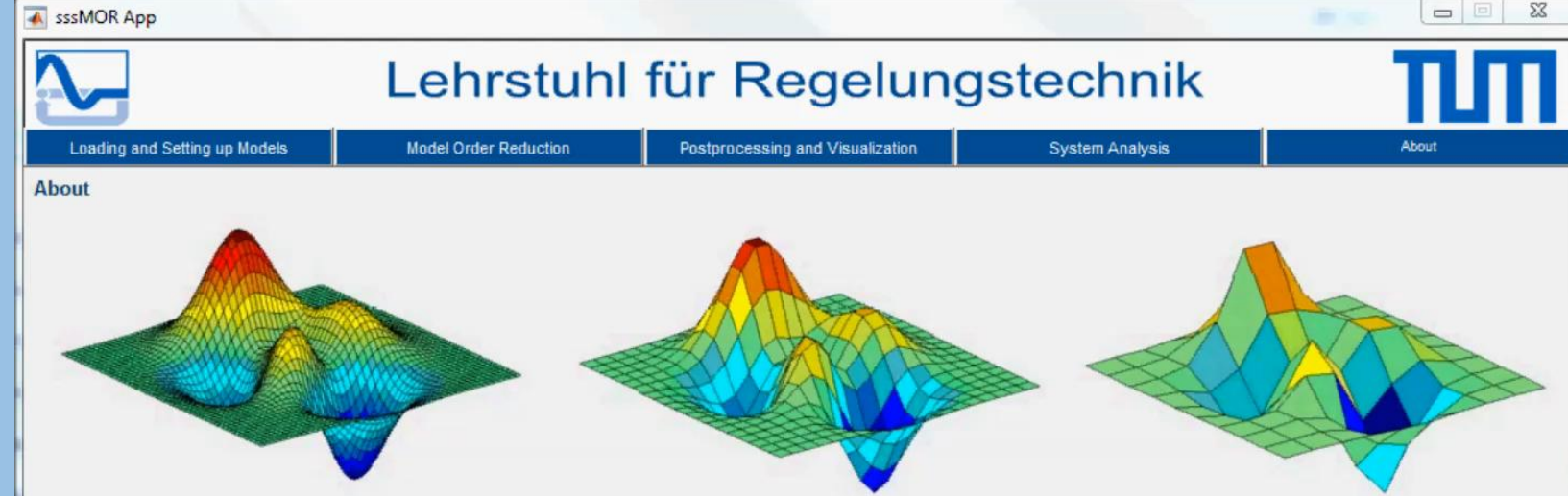

Welcome to the sssinOR App

developed at the Chair of Automatic Control, TU München

Loading and Setting up Models

Model Order Reduction

Postprocessing and Visualization

System Analysis
Load, create and save models

Reduce models

Plot Impulse Response, Step Response,

Bode Diagram, Frequency Response and Pole-Zero Map

Analyse models
The sss MOR App is primarily implemented for demonstration and educational purposes and does not explot the full functionality of the sssMOR toolbox.

Further information avaliable under: hHtps:/Www it mw tum de/7sssmor

Version 1.0 

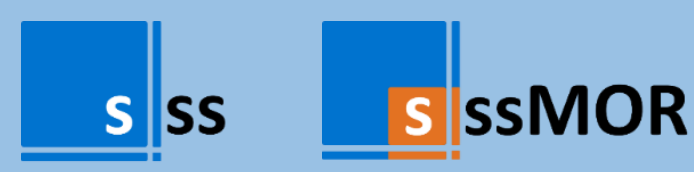

Comprehensive documentation with examples and references

\section{sssMOR App}

graphical user interface
A MATLAB R2015b - academic use

\begin{tabular}{|c|c|c|c|c|c|}
\hline HOME & PLOTS & APPS & a & Search Documentation & \\
\hline
\end{tabular}

\section{Name Git $f_{x}>>1$}

\pm extras

⿴囗十 MOR

(t) 555 completely free and open source (contributions welcome)

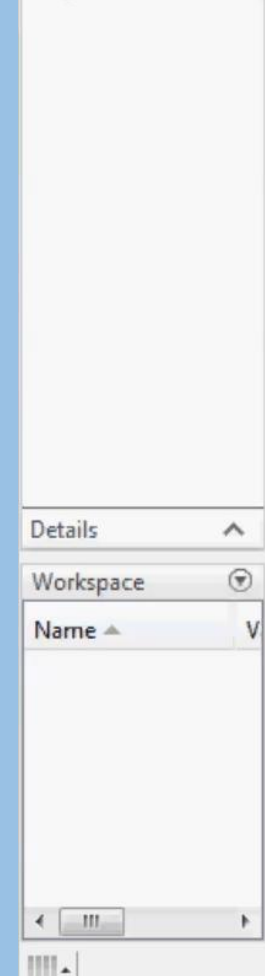

